\title{
Exploiting $C P$ asymmetries in rare charm decays
}

\author{
Rigo Bause ${ }^{*}{ }^{*}$ Hector Gisbert $\odot,^{\dagger}$ Marcel Golz $\odot,{ }^{\dagger}$ and Gudrun Hiller ${ }^{\S}$ \\ Fakultät für Physik, TU Dortmund, Otto-Hahn-Str. 4, D-44221 Dortmund, Germany
}

(Received 9 April 2020; accepted 12 May 2020; published 3 June 2020)

\begin{abstract}
We analyze patterns from $\mathrm{CP}$-violating new physics (NP) in hadronic and semileptonic rare charm $|\Delta c|=|\Delta u|=1$ transitions. Observation of direct $C P$ violation in hadronic decays, as in $\Delta A_{C P}$, provides opportunities for $c \rightarrow u \ell^{+} \ell^{-}, \ell=e, \mu$ transitions, and vice versa. For the concrete case of flavorful, anomalyfree $Z^{\prime}$-models a NP-interpretation of $\Delta A_{C P}$ suggests measurable $C P$ asymmetries in semileptonic decays such as $D \rightarrow \pi \ell^{+} \ell^{-}$or $D \rightarrow \pi \pi \ell^{+} \ell^{-}$. Conversely, an observation of $C P$ violation in $c \rightarrow u e^{+} e^{-}$or $c \rightarrow u \mu^{+} \mu^{-}$decays supports a NP-interpretation of $\Delta A_{C P}$. Flavorful $U(1)^{\prime}$-extensions provide explicit $\mathrm{U}$-spin and isospin breaking which can be probed in patterns of hadronic decays of charm mesons. We work out signatures for $C P$ asymmetries in $D^{0} \rightarrow \pi^{+} \pi^{-}, D^{0} \rightarrow K^{+} K^{-}$and $D^{0} \rightarrow \pi^{0} \pi^{0}$, $D^{+} \rightarrow \pi^{+} \pi^{0}$ decays, which can be probed in the future at LHCb and Belle II and provide further informative cross checks.
\end{abstract}

DOI: 10.1103/PhysRevD.101.115006

\section{INTRODUCTION}

Suppressions of standard model (SM) amplitudes due to accidental symmetries provide useful directions for searches for new physics (NP). Among the salient features of $|\Delta c|=|\Delta u|=1$ transitions within the SM are a strong Glashow-Iliopoulos-Maiani (GIM)-suppression and small $C P$ violation. Hierarchies of the Cabibbo-KobayashiMaskawa (CKM) matrix $V$ suggest SM $C P$ violation at the order of $\operatorname{Im}\left(V_{c b}^{*} V_{u b} /\left(V_{c s}^{*} V_{u s}\right)\right) \sim 7 \times 10^{-4}$, somewhat below LHCb's observation of $C P$ violation in charm [1]

$$
\begin{aligned}
\Delta A_{C P} & =A_{C P}\left(K^{+} K^{-}\right)-A_{C P}\left(\pi^{+} \pi^{-}\right) \\
& =(-15.4 \pm 2.9) \times 10^{-4},
\end{aligned}
$$

where

$$
A_{C P}(f)=\frac{\Gamma\left(D^{0} \rightarrow f\right)-\Gamma\left(\bar{D}^{0} \rightarrow f\right)}{\Gamma\left(D^{0} \rightarrow f\right)+\Gamma\left(\bar{D}^{0} \rightarrow f\right)},
$$

and the corresponding world average [2]

$$
\Delta A_{C P}^{\mathrm{HFLAV}}=(-16.4 \pm 2.8) \times 10^{-4} .
$$

\footnotetext{
*rigo.bause@tu-dortmund.de hector.gisbert@tu-dortmund.de *marcel.golz@tu-dortmund.de

§ghiller@physik.uni-dortmund.de
}

Published by the American Physical Society under the terms of the Creative Commons Attribution 4.0 International license. Further distribution of this work must maintain attribution to the author(s) and the published article's title, journal citation, and DOI. Funded by SCOAP ${ }^{3}$.
While this leaves room for NP, due to the sizable uncertainties of hadronic $D$-decays, Eqs. (1) and (3) provide no clear-cut sign of NP. On the other hand, $\Delta A_{C P}$ as large as the permille level is nontrivial to achieve in concrete models of NP. Correlations with other observables in charm and the down-quark sector exist, which are subject to partly very strong flavor constraints. For recent works, see Refs. [3-12]. Turning this around, the study of patterns using different sectors can hence disfavor or support a particular $\Delta A_{C P}$ interpretation, and vice versa.

In this work we pursue a global analysis of $C P$ asymmetries in rare hadronic and semileptonic charm decays. Our focus is on NP patterns induced by four-fermion operators. Links via dipole operators between hadronic and semileptonic $C P$ asymmetries in $D \rightarrow \pi \ell^{+} \ell^{-}$decays have been pointed out by Ref. [13]. We work out predictions and correlations for anomalyfree $Z^{\prime}$-extensions of the SM with generation-dependent $U(1)^{\prime}$-charges, see Refs. [14-20] for recent phenomenological works. Flavorful charges can give rise to explicit isospin and U-spin breaking effects. It is our goal to work out corresponding experimental signatures for hadronic charm decays, exploiting yet another SM null test strategy in charm [21].

This paper is organized as follows: In Sec. II we briefly review $C P$ violation in hadronic $D$-decays, $D$-mixing and semileptonic $c \rightarrow u \ell^{+} \ell^{-}$transitions. In Sec. III we analyze effects of anomalyfree $U(1)^{\prime}$-extensions with generationdependent charges in hadronic 2-body $D$-decays and how $D$-mixing constraints can be evaded to address $\Delta A_{C P}$. Patterns among $C P$ asymmetries in $D^{0} \rightarrow \pi^{+} \pi^{-}$, $D^{0} \rightarrow K^{+} K^{-}, D^{0} \rightarrow \pi^{0} \pi^{0}$ and $D^{+} \rightarrow \pi^{+} \pi^{0}$ decays are worked out in Sec. IV. Correlations with $C P$ asymmetries in rare semileptonic decays are studied in Sec. V. We 
conclude in Sec. VI. Auxiliary information is given in several appendices.

\section{CP PHENOMENOLOGY IN CHARM}

We review $C P$ violation in hadronic $D$-decays (Sec. II A), $D$-mixing (Sec. II B) and semileptonic $c \rightarrow u \ell^{+} \ell^{-}$processes (Sec. II C).

\section{A. Direct $C P$ violation in $D^{0} \rightarrow \pi^{+} \pi^{-}, K^{+} K^{-}$}

The single-Cabibbo-suppressed (SCS) $D^{0}\left(\bar{D}^{0}\right)$ decay amplitudes $\mathcal{A}_{f}\left(\overline{\mathcal{A}}_{f}\right)$ to $C P$ eigenstates $f$ can be written as

$$
\begin{aligned}
& \mathcal{A}_{f}=\mathcal{A}_{f}^{T} \mathrm{e}^{\mathrm{i} \phi_{f}^{T}}\left[1+r_{f} \mathrm{e}^{\mathrm{i}\left(\delta_{f}+\phi_{f}\right)}\right], \\
& \overline{\mathcal{A}}_{f}=\eta_{C P} \mathcal{A}_{f}^{T} \mathrm{e}^{-\mathrm{i} \phi_{f}^{T}}\left[1+r_{f} \mathrm{e}^{\mathrm{i}\left(\delta_{f}-\phi_{f}\right)}\right],
\end{aligned}
$$

where $\eta_{C P}= \pm 1$ is the $C P$ eigenvalue of $f$. The dominant SCS "tree" amplitude in the SM is denoted by $\mathcal{A}_{f}^{T} \mathrm{e}^{ \pm \mathrm{i} \phi_{f}^{T}}$, and $r_{f}$ parametrizes the relative magnitude of all subleading amplitudes. Inserting Eqs. (4) into Eq. (2), in the limit of $r_{f} \ll 1$, yields

$$
A_{C P}(f)=-2 r_{f} \sin \delta_{f} \sin \phi_{f}+\mathcal{O}\left(r_{f}^{2}\right),
$$

requiring both strong $\left(\delta_{f}\right)$ and weak $\left(\phi_{f}\right)$ relative phases for a nonvanishing direct $C P$ asymmetry. Beyond the SM the SCS $D^{0}$ decay amplitude can be written as

$$
\mathcal{A}_{f}=\sum_{q=d, s, b} \lambda_{q}\left(\mathcal{A}_{f}^{q}\right)_{\mathrm{SM}}+\mathcal{A}_{f}^{\mathrm{NP}},
$$

where the first term corresponds to the SM contribution with CKM-factors $\lambda_{q}=V_{c q}^{*} V_{u q}$ made explicit, and the second term accounts for NP. Using CKM unitarity $\lambda_{d}+\lambda_{s}+\lambda_{b}=0$ and writing for the final states $K^{+} K^{-}$ and $\pi^{+} \pi^{-}$in the subscripts $f=K$ and $f=\pi$, respectively, one finds

$$
\begin{aligned}
\mathcal{A}_{K(\pi)}= & \lambda_{s(d)}\left(\mathcal{A}_{K(\pi)}^{s(d)}-\mathcal{A}_{K(\pi)}^{d(s)}\right)_{\mathrm{SM}} \\
& +\lambda_{b}\left(\mathcal{A}_{K(\pi)}^{b}-\mathcal{A}_{K(\pi)}^{d(s)}\right)_{\mathrm{SM}}+\mathcal{A}_{K(\pi)}^{\mathrm{NP}} .
\end{aligned}
$$

Here, the first term is the SCS contribution and the second one corresponds to "penguin" contributions with small Wilson coefficients which are strongly CKM-suppressed with respect to the SCS one by $\lambda_{b} / \lambda_{s, d}$. The last term $\mathcal{A}_{K(\pi)}^{\mathrm{NP}}$ encodes NP contributions. Using Eqs. (4), (5) and (7), we obtain

$$
\Delta A_{C P}=\Delta A_{C P}^{\mathrm{SM}}-\frac{2}{\left|\lambda_{s, d}\right|} \Delta r^{\mathrm{NP}},
$$

where $^{1}$

\footnotetext{
${ }^{1}$ The plus sign between the pion and kaon amplitudes in Eq. (9) comes from $\lambda_{d}=-\lambda_{s}+\mathcal{O}\left(\lambda_{b}\right)$.
}

$$
\Delta r^{\mathrm{NP}}=r_{K} \sin \delta_{K} \sin \phi_{K}+r_{\pi} \sin \delta_{\pi} \sin \phi_{\pi},
$$

and

$$
r_{K}=\frac{\mathcal{A}_{K}^{\mathrm{NP}}}{\left(\mathcal{A}_{K}^{s}-\mathcal{A}_{K}^{d}\right)_{\mathrm{SM}}}, \quad r_{\pi}=\frac{\mathcal{A}_{\pi}^{\mathrm{NP}}}{\left(\mathcal{A}_{\pi}^{d}-\mathcal{A}_{\pi}^{s}\right)_{\mathrm{SM}}},
$$

and $r_{\pi, K} \ll 1$. The strong phases $\delta_{\pi, K}$ are associated with the NP amplitudes. Since we are interested in maximal NP contributions, we employ in our numerical analysis $\sin \delta_{\pi, K} \sim 1$. Note, there is a priori no information on the sign of $\Delta r^{\mathrm{NP}}$ as it depends on products of strong and weak phases. The branching ratios of the $D \rightarrow f$ modes are dominated by their respective SM contributions. We can therefore extract $\left|\left(\mathcal{A}_{K(\pi)}^{s(d)}-\mathcal{A}_{K(\pi)}^{d(s)}\right)_{\mathrm{SM}}\right|$ from data, see Appendix A for details.

\section{B. $C P$ violation in $D^{0}-\bar{D}^{\mathbf{0}}$ mixing}

Here we consider constraints from charm meson mixing. The $D^{0}-\bar{D}^{0}$ transition amplitude can be written as

$$
\left\langle D^{0}\left|\mathcal{H}_{\mathrm{eff}}^{\Delta c=2}\right| \bar{D}^{0}\right\rangle=M_{12}-\frac{\mathrm{i}}{2} \Gamma_{12},
$$

which can be parametrized in terms of the following physical quantities

$x_{12}=2 \frac{\left|M_{12}\right|}{\Gamma}, \quad y_{12}=\frac{\left|\Gamma_{12}\right|}{\Gamma}, \quad \phi_{12}=\arg \left(\frac{M_{12}}{\Gamma_{12}}\right)$.

Here, $x_{12}$ and $y_{12}$ are $C P$ conserving, while $\phi_{12}$ is a phase difference that results in $C P$ violation in mixing. A global fit from the HFLAV collaboration [2] results in

$$
\begin{aligned}
x_{12} & \in[0.22,0.63] \%, \\
y_{12} & \in[0.50,0.75] \%, \\
\phi_{12} & \in\left[-2.5^{\circ}, 1.8^{\circ}\right] .
\end{aligned}
$$

In absence of a sufficiently controlled SM prediction of the mixing parameters, we require the NP contributions to saturate the current world averages (13),

$$
\begin{gathered}
x_{12}^{\mathrm{NP}} \leq x_{12}, \quad x_{12}^{\mathrm{NP}} \sin \phi_{12}^{\mathrm{NP}} \leq x_{12} \sin \phi_{12} . \\
\text { C. } \boldsymbol{C P} \text { violation in } \boldsymbol{c} \rightarrow \boldsymbol{u} \boldsymbol{\ell}^{+} \boldsymbol{\ell}^{-}
\end{gathered}
$$

$C P$ violation in semileptonic rare charm decays arises from complex-valued Wilson coefficients $C_{i}^{\ell \ell}, C_{i}^{\ell \ell l}$ in the effective Hamiltonian [18],

$\mathcal{H}_{\text {eff }} \supset-\frac{4 G_{F}}{\sqrt{2}} \frac{\alpha_{e}}{4 \pi} \sum_{i=9,10}\left(C_{i}^{\ell \ell} O_{i}^{\ell \ell}+C_{i}^{\ell \ell \prime} O_{i}^{\ell \ell \prime}\right)+$ H.c.,

with the operators 


$$
\begin{aligned}
O_{9}^{\ell \ell(I)} & =\left(\bar{u}_{L(R)} \gamma_{\mu} c_{L(R)}\right)\left(\bar{\ell} \gamma^{\mu} \ell\right), \\
O_{10}^{\ell \ell(\prime)} & =\left(\bar{u}_{L(R)} \gamma_{\mu} c_{L(R)}\right)\left(\bar{\ell} \gamma^{\mu} \gamma_{5} \ell\right) .
\end{aligned}
$$

Here, $\alpha_{e}$ denotes the fine structure constant, $G_{F}$ is Fermi's constant and $L=\left(1-\gamma_{5}\right) / 2, R=\left(1+\gamma_{5}\right) / 2$ are chiral projectors. $C P$ violation has not been observed in semileptonic $|\Delta c|=|\Delta u|=1$ decays yet. Available measurements for $C P$ asymmetries in rare semileptonic charm decays are at the level of few to $\mathcal{O}(10) \%$ [22], which is close to possible NP effects $[13,18,21]$.

Branching ratio and high $-p_{T}$ data imply the following constraints, barring cancellations [23,24]

$$
\left|C_{9,10}^{\mu \mu(\prime)}\right| \lesssim 1, \quad\left|C_{9,10}^{e e(\prime)}\right| \lesssim 3
$$

stronger for muons than for electrons.

\section{A FLAVORFUL $Z^{\prime}$ IN CHARM}

We work out NP-effects in charm from anomalyfree $U(1)^{\prime}$-extensions of the SM with fermion charges $F_{\psi_{i}}$ that depend on the generation, $i=1,2$, 3. Specifically, SM fermion multiplets plus possibly right-handed neutrinos $\psi=Q, u, d, L, e, \nu$ in representations of $S U(3)_{C} \times$ $S U(2)_{L} \times U(1)_{Y} \times U(1)^{\prime}$ can be characterized, in that order, as

$$
\begin{aligned}
Q_{i} & =\left(3,2,1 / 6, F_{Q_{i}}\right), & u_{i} & =\left(3,1,2 / 3, F_{u_{i}}\right), \\
d_{i} & =\left(3,1,-1 / 3, F_{d_{i}}\right), & L_{i} & =\left(1,2,-1 / 2, F_{L_{i}}\right), \\
e_{i} & =\left(1,1,-1, F_{e_{i}}\right), & \nu_{i} & =\left(1,1,0, F_{\nu_{i}}\right) .
\end{aligned}
$$

Concrete models with $F_{\psi_{i}}$-assignments that fulfill the anomaly-cancellation conditions and induce $c \rightarrow u$ flavor changing neutral currents (FCNCs) are given in Table I. Related models (models 1 to 8 ) have been studied previously in the context of semileptonic rare charm decays in Ref. [18], to which we refer for further details. The models in Table I satisfy $\sum_{i=1}^{3}\left(F_{Q_{i}}-F_{L_{i}}+2 F_{u_{i}}-F_{d_{i}}-F_{e_{i}}\right)=0$ and therefore avoid kinetic mixing at one-loop [25].
In Sec. III A we discuss couplings of the fermions to the $Z^{\prime}$-boson, which arises from the $U(1)^{\prime}$-group. We assume the $Z^{\prime}$ to have a mass $M_{Z^{\prime}}$ of the electroweak scale or heavier. We discuss the induced $c \rightarrow u$ four-quark operators and Wilson coefficients in Sec. III B. In Sec. III C we discuss how to bypass constraints from $D^{0}-\bar{D}^{0}$ mixing. We work out predictions for $\Delta A_{C P}$ in Sec. III D.

\section{A. $Z^{\prime}-$ FCNCs}

The $Z^{\prime}$-couplings relevant to charm FCNCs can be written as

$$
\begin{aligned}
\mathcal{L}_{Z^{\prime}} \supset & \left(g_{L}^{u c} \bar{u}_{L} \gamma^{\mu} c_{L} Z_{\mu}^{\prime}+g_{R}^{u c} \bar{u}_{R} \gamma^{\mu} c_{R} Z_{\mu}^{\prime}+\text { H.c. }\right) \\
& +g_{L}^{d} \bar{d}_{L} \gamma^{\mu} d_{L} Z_{\mu}^{\prime}+g_{R}^{d} \bar{d}_{R} \gamma^{\mu} d_{R} Z_{\mu}^{\prime} \\
& +g_{L}^{s} \bar{s}_{L} \gamma^{\mu} s_{L} Z_{\mu}^{\prime}+g_{R}^{s} \bar{s}_{R} \gamma^{\mu} s_{R} Z_{\mu}^{\prime} \\
& +g_{L}^{\ell \ell} \bar{\ell}_{L} \gamma^{\mu} \ell_{L} Z_{\mu}^{\prime}+g_{R}^{\ell \ell} \bar{\ell}_{R} \gamma^{\mu} \ell_{R} Z_{\mu}^{\prime}
\end{aligned}
$$

with $\ell=e, \mu, \tau$. The flavor diagonal couplings $g_{L, R}^{d, s}$ and $g_{L, R}^{\ell \ell}$ are given as the $U(1)^{\prime}$-gauge coupling $g_{4}$ times the associated charge $F_{\psi}$.

The $|\Delta c|=|\Delta u|=1$ FCNC couplings $g_{L, R}^{u c}$ are generated via rotations from the gauge to the mass basis, and are in general complex-valued. Four different unitary rotations exist in the quark sector, corresponding to the left-handed (LH) and right-handed (RH) ones both for up- and down-type quarks. The product of LH up- and down-type rotations gives the CKM-matrix. In order to evade the severe constraints in the kaon sector, we assume the CKMmatrix to predominantly stem from the LH up-type rotation, implying

$$
g_{L}^{u c} \approx g_{4} \lambda_{\mathrm{CKM}} \Delta F_{L}, \quad \Delta F_{L}=F_{Q_{2}}-F_{Q_{1}},
$$

\begin{tabular}{|c|c|c|c|c|c|c|c|c|c|c|c|c|c|c|c|c|c|c|}
\hline \multirow{2}{*}{$\frac{\text { Model }}{2}$} & \multicolumn{3}{|c|}{$F_{Q_{i}}$} & \multicolumn{3}{|c|}{$F_{u_{i}}$} & \multicolumn{3}{|c|}{$F_{d_{i}}$} & \multicolumn{3}{|c|}{$F_{L_{i}}$} & \multicolumn{3}{|c|}{$F_{e_{i}}$} & \multicolumn{3}{|c|}{$F_{\nu_{i}}$} \\
\hline & 3 & 3 & -6 & -8 & 4 & 4 & -10 & 10 & 0 & -6 & 5 & 1 & 0 & 0 & 0 & 0 & 0 & 0 \\
\hline 4 & -1 & -1 & 2 & -1 & 2 & -1 & 0 & 0 & 0 & -1 & 1 & 0 & -2 & 2 & 0 & -2 & -1 & 3 \\
\hline 5 & -1 & -1 & 2 & -1 & 2 & -1 & 2 & -1 & -1 & -1 & 1 & 0 & -1 & 1 & 0 & 0 & 0 & 0 \\
\hline 9 & 0 & 0 & 0 & -11 & -2 & 13 & 7 & 7 & -14 & -8 & 3 & 5 & -6 & 16 & -10 & 0 & 0 & 0 \\
\hline 10 & 0 & 0 & 0 & -13 & 6 & 7 & -1 & -14 & 15 & -15 & 15 & 0 & -14 & 18 & -4 & 0 & 0 & 0 \\
\hline $10 \mu$ & 0 & 0 & 0 & -13 & 6 & 7 & -1 & -14 & 15 & -15 & 0 & 15 & -14 & -4 & 18 & 0 & 0 & 0 \\
\hline
\end{tabular}

where $\lambda_{\text {CKM }} \sim 0.2$ denotes the Wolfenstein parameter and we used $\lambda_{\mathrm{CKM}} \ll 1$. In contrast, the RH rotation is a priori unconstrained and induces

$$
g_{R}^{u c}=g_{4} \sin \theta_{u} \cos \theta_{u} e^{\mathrm{i} \phi_{R}} \Delta F_{R},
$$

TABLE I. Sample solutions of an anomalyfree $U(1)^{\prime}$-extension of the $\mathrm{SM}+3 \nu_{R}$ with $F_{Q_{1}}=F_{Q_{2}}$. Models 2,4 and 5 are taken from Ref. [18]. Models 9 and 10 feature $F_{Q_{i}}=0$. In general, the ordering of generations is arbitrary due to permutation invariance. However, our analysis explicitly uses the ordering stated here, that is, the $i$ th entry corresponds to the $i$ th generation. Model $10 \mu$ is the same as model 10 with the smallest lepton-coupling to muons. 
where $\theta_{u}$ is the up-charm mixing angle for the up-quark singlets, $\Delta F_{R}=F_{u_{2}}-F_{u_{1}}$ and $\phi_{R}$ the corresponding $C P$-phase.

\section{B. Four-fermion operators and matching}

Generation-dependent quark-couplings result in additional operators in the effective weak Hamiltonian beyond the ones considered usually, i.e., Ref. [26]. At the scale $m_{b}<\mu<\mu_{\mathrm{EWK}}$,

$$
\mathcal{H}_{\mathrm{eff}}^{|\Delta \mathrm{c}|=1} \supset \frac{G_{F}}{\sqrt{2}} \sum_{i} \tilde{C}_{i}^{(\prime)} \tilde{Q}_{i}^{(\prime)}+\text { H.c. }
$$

with the new operators

$$
\begin{gathered}
\tilde{Q}_{7}=(\bar{u} c)_{V-A} \sum_{q} F_{u_{i}, d_{i}}(\bar{q} q)_{V+A}, \\
\tilde{Q}_{7}^{\prime}=(\bar{u} c)_{V+A} \sum_{q} F_{Q_{i}}(\bar{q} q)_{V-A}, \\
\tilde{Q}_{8}=\left(\bar{u}_{\alpha} c_{\beta}\right)_{V-A} \sum_{q} F_{u_{i}, d_{i}}\left(\bar{q}_{\beta} q_{\alpha}\right)_{V+A}, \\
\tilde{Q}_{8}^{\prime}=\left(\bar{u}_{\alpha} c_{\beta}\right)_{V+A} \sum_{q} F_{Q_{i}}\left(\bar{q}_{\beta} q_{\alpha}\right)_{V-A}, \\
\tilde{Q}_{9}=(\bar{u} c)_{V-A} \sum_{q} F_{Q_{i}}(\bar{q} q)_{V-A}, \\
\tilde{Q}_{9}^{\prime}=(\bar{u} c)_{V+A} \sum_{q} F_{u_{i}, d_{i}}(\bar{q} q)_{V+A}, \\
\tilde{Q}_{10}=\left(\bar{u}_{\alpha} c_{\beta}\right)_{V-A} \sum_{q} F_{Q_{i}}\left(\bar{q}_{\beta} q_{\alpha}\right)_{V-A}, \\
\tilde{Q}_{10}^{\prime}=\left(\bar{u}_{\alpha} c_{\beta}\right)_{V+A} \sum_{q} F_{u_{i}, d_{i}}\left(\bar{q}_{\beta} q_{\alpha}\right)_{V+A},
\end{gathered}
$$

where $(V \pm A)$ refers to the Dirac structures $\gamma_{\mu}\left(1 \pm \gamma_{5}\right)$, $q=u, c, d, s, b$ and $\alpha, \beta$ are the color indices. The strength of these operators is given by their respective Wilson coefficients $\tilde{C}_{i}, \tilde{C}_{i}^{\prime}$ which depend on both heavy masses and weak phases responsible for $C P$-violating phenomena. The Wilson coefficients induced by the Lagrangian (20) read

$$
\begin{aligned}
& \tilde{C}_{7}\left(M_{Z^{\prime}}\right)=\tilde{C}_{9}\left(M_{Z^{\prime}}\right)=\frac{\sqrt{2}}{G_{F}} g_{L}^{u c} \frac{g_{4}}{4 M_{Z^{\prime}}^{2}}, \\
& \tilde{C}_{7}^{\prime}\left(M_{Z^{\prime}}\right)=\tilde{C}_{9}^{\prime}\left(M_{Z^{\prime}}\right)=\frac{\sqrt{2}}{G_{F}} g_{R}^{u c} \frac{g_{4}}{4 M_{Z^{\prime}}^{2}}, \\
& \tilde{C}_{8}^{(\prime)}\left(M_{Z^{\prime}}\right)=\tilde{C}_{10}^{(\prime)}\left(M_{Z^{\prime}}\right)=0 .
\end{aligned}
$$

They are evolved from $M_{Z^{\prime}}$ to $m_{c}$ using the renormalization group equations (RGEs) with top and bottom quarks integrated out at their respective threshold scales. Finite values of $\tilde{C}_{8}^{(\prime)}$ and $\tilde{C}_{10}^{(\prime)}$ arise from the RGE mixing at the charm mass scale, see Appendix B for details.

\section{C. $D^{\mathbf{0}}-\bar{D}^{\mathbf{0}}$ mixing constraints}

Rare $|\Delta c|=|\Delta u|=1$ decays are induced in the $Z^{\prime}$-models by operators with coefficients proportional to $g_{L}^{u c}$ or $g_{R}^{u c}$ in Eq. (32). These couplings induce at second order $D^{0}-\bar{D}^{0}$ mixing (32), and are constrained as

$$
\left|\left(g_{L}^{u c}\right)^{2}+\left(g_{R}^{u c}\right)^{2}-X g_{L}^{u c} g_{R}^{u c}\right| \lesssim 6 \times 10^{-7}\left(\frac{M_{Z^{\prime}}}{\mathrm{TeV}}\right)^{2},
$$

with $X \sim 20$ for $M_{Z^{\prime}}$ in the TeV range [18]. This constraint on $x_{12}$ can be evaded if both $g_{L}^{u c}$ and $g_{R}^{u c}$ are present, for either $g_{L}^{u c} \sim X g_{R}^{u c}$ or $g_{L}^{u c} \sim 1 / X g_{R}^{u c}$. However, in these cases the $C P$ phases have to be aligned $\operatorname{Arg}\left(g_{L}^{u c}\right) \sim \operatorname{Arg}\left(g_{R}^{u c}\right)$ to fulfill Eq. (33). As kaon constraints force $\operatorname{Arg}\left(g_{L}^{u c}\right)$ to be SM-like, $C P$-violating effects in charm become negligible.

We therefore choose $g_{L}^{u c}=0$, which can be achieved with $\Delta F_{L}=0$. The models in Table I satisfy for this reason $F_{Q_{1}}=F_{Q_{2}}$. Consequently, we focus on FCNCs in the upsinglet sector (22), that is, $g_{R}^{u c} \neq 0$ and complex.

If there is a single coupling only, the above mixing constraint on $x_{12}$ becomes

$$
\left|g_{A}^{u c}\right| \lesssim 8 \times 10^{-4}\left(\frac{M_{Z^{\prime}}}{\mathrm{TeV}}\right), \quad A=L, R
$$

The even tighter constraint (14) for $C P$-violating couplings on $x_{12} \sin \phi_{12}$ can be bypassed for $\operatorname{Arg}\left(g_{R}^{u c}\right)=\phi_{R}$ around $\pi / 2$ (or $3 \pi / 2$ ), as the $C P$ phase of the mixing amplitude is twice the one of the $|\Delta c|=|\Delta u|=1$ FCNC [26]. The contributions to $\Delta A_{C P}$ become maximal while simultaneously mixing constraints are satisfied. This interplay of $\phi_{R}$ versus the coupling $g_{4} / M_{Z^{\prime}}\left(\mathrm{TeV}^{-1}\right)$ for model 2 and fixed $\theta_{u}=1 \times 10^{-4}$ is illustrated in Fig. 1. The red (hatched) area corresponds to the $D^{0}-\bar{D}^{0}$ mixing constraints on the imaginary part $x_{12} \sin \phi_{12}$ (absolute value $x_{12}$ ). $Z^{\prime}$-induced values of $\Delta A_{C P}$ are shown in green. Indeed the region around $\phi_{R} \sim \pi / 2$ is viable and can induce $\Delta A_{C P}^{\mathrm{NP}} \sim 10^{-3}$.

\section{D. $Z^{\prime}$-effects for $\Delta A_{C P}$}

Taking into account the running from $M_{Z^{\prime}}$ to $m_{c}$, details of which are given in Appendix $\mathrm{B}$, we find that $\Delta A_{C P}$ can be written as

$$
\Delta A_{C P}^{\mathrm{NP}}=A_{C P}^{\mathrm{NP}}\left(K^{+} K^{-}\right)-A_{C P}^{\mathrm{NP}}\left(\pi^{+} \pi^{-}\right),
$$

with 


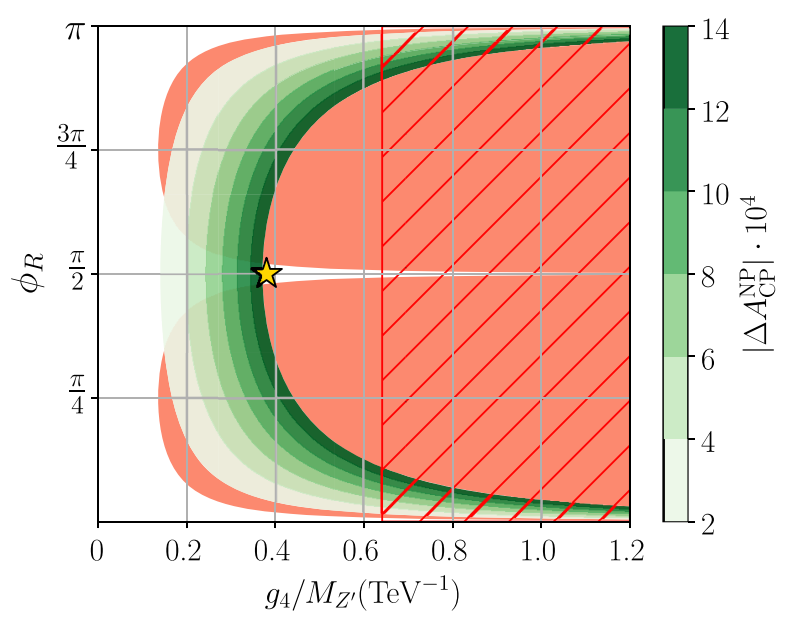

FIG. 1. $\left|\Delta A_{C P}^{\mathrm{NP}}\right|$ (green bands) versus $D^{0}-\bar{D}^{0}$ mixing exclusion regions (14) on the imaginary part $x_{12} \sin \phi_{12}$ (red area) and the absolute value $x_{12}$ (red hatched area) in the $\phi_{R}-g_{4} / M_{Z^{\prime}}\left(\mathrm{TeV}^{-1}\right)$ plane for $\theta_{u}=1 \times 10^{-4}$. $F_{\psi}$-charges are as in model 2 , see Table I. The golden star indicates a benchmark point (40), see text for details.

$$
\begin{aligned}
A_{C P}^{\mathrm{NP}}\left(K^{+} K^{-}\right) & \sim \frac{g_{4}^{2}}{M_{Z^{\prime}}^{2}} \theta_{u} \Delta F_{R}\left[c_{K} F_{Q_{2}}+d_{K} F_{d_{2}}\right] \\
A_{C P}^{\mathrm{NP}}\left(\pi^{+} \pi^{-}\right) & \sim \frac{g_{4}^{2}}{M_{Z^{\prime}}^{2}} \theta_{u} \Delta F_{R}\left[c_{\pi} F_{Q_{1}}+d_{\pi} F_{d_{1}}\right]
\end{aligned}
$$

where

$$
\begin{array}{rlrl}
c_{K} & =\frac{\chi_{K}}{a_{K}} r_{1}\left(m_{c}, M_{Z^{\prime}}\right), & c_{\pi} & =-\frac{\chi_{\pi}}{a_{\pi}} r_{1}\left(m_{c}, M_{Z^{\prime}}\right), \\
d_{K}=\frac{1}{a_{K}} r_{2}\left(m_{c}, M_{Z^{\prime}}\right), & d_{\pi}=-\frac{1}{a_{\pi}} r_{2}\left(m_{c}, M_{Z^{\prime}}\right) .
\end{array}
$$

As explained in the previous Sec. III C, we analyze models with $g_{L}^{u c}=0$ and $\operatorname{Im}\left(g_{R}^{u c}\right)$ large. In Eq. (36) we use $\sin \delta_{\pi, K} \sin \phi_{R} \sim 1$ and anticipated $\theta_{u} \ll 1$. The parameters $c_{K, \pi}$ and $d_{K, \pi}$ depend on the chiral factors $\chi_{K, \pi}$ at the charm scale, the LO QCD running functions $r_{1,2}\left(m_{c}, M_{Z^{\prime}}\right)$ and the tree-level contributions $a_{K, \pi}$, which are determined experimentally. Further details can be found in Appendixes A-D. Numerical values of $c_{K, \pi}$ and $d_{K, \pi}$ for different $Z^{\prime}$ masses are displayed in Table II.

In Fig. 2 we show sizable $Z^{\prime}$-contributions to $\Delta A_{C P}^{\mathrm{NP}}$ and $D^{0}-\bar{D}^{0}$ mixing constraints (red area) in the plane of $g_{4} / M_{Z^{\prime}}\left(\mathrm{TeV}^{-1}\right)$ and the parameter $\Delta \tilde{F}_{R}=\Delta F_{R} \theta_{u}$ for models 2, 5, 9 and $10(\mu)$. The corresponding plot of model 4 is not given in Fig. 2 because it exhibits very similar bands as model 5 due to identical $F_{Q_{1,2}}$ and $\Delta F_{R}$, as shown in Table I. Constraints from branching ratios of (semi-) muonic $D$-decays (dash-dotted and dotted lines), here for $g_{L}^{u c}=0,[18]$
TABLE II. Parameters $c_{K, \pi}, d_{K, \pi}$ and $d_{\pi^{\prime}, \pi^{0}}$ in $(\mathrm{TeV})^{2}$ as defined in Eq. (37) and Eq. (53), respectively, for different $Z^{\prime}$ masses.

\begin{tabular}{lrrrrr}
\hline \hline$M_{Z^{\prime}}[\mathrm{TeV}]$ & \multicolumn{1}{c}{2} & \multicolumn{1}{c}{4} & \multicolumn{1}{c}{6} & \multicolumn{1}{c}{8} & \multicolumn{1}{c}{10} \\
\hline$c_{K}$ & 1.133 & 1.217 & 1.266 & 1.302 & 1.330 \\
$d_{K}$ & -0.046 & -0.054 & -0.058 & -0.061 & -0.063 \\
$c_{\pi}$ & -1.446 & -1.553 & -1.616 & -1.661 & -1.698 \\
$d_{\pi}$ & 0.058 & 0.068 & 0.074 & 0.077 & 0.080 \\
$d_{\pi^{\prime}}$ & 0.071 & 0.083 & 0.090 & 0.094 & 0.098 \\
$d_{\pi^{0}}$ & 0.077 & 0.090 & 0.097 & 0.102 & 0.106 \\
\hline \hline
\end{tabular}

$$
\begin{aligned}
& \left|g_{R}^{u c}\right| \sqrt{\left(g_{L}^{\mu \mu}\right)^{2}+\left(g_{R}^{\mu \mu}\right)^{2}} \\
& =g_{4}^{2}\left|\Delta \tilde{F}_{R}\right| \sqrt{F_{L_{2}}^{2}+F_{e_{2}}^{2}} \lesssim 0.04\left(\frac{M_{Z^{\prime}}}{\mathrm{TeV}}\right)^{2}, \\
& \left|g_{R}^{u c}\left(g_{L}^{\mu \mu}-g_{R}^{\mu \mu}\right)\right| \\
& \quad=g_{4}^{2}\left|\Delta \tilde{F}_{R}\left(F_{L_{2}}-F_{e_{2}}\right)\right| \lesssim 0.03\left(\frac{M_{Z^{\prime}}}{\mathrm{TeV}}\right)^{2},
\end{aligned}
$$

start to be competitive with mixing constraints close to the nonperturbativity region (black region). This is particularly relevant for model 9 and 10, which exhibit large couplings to leptons. To evade the muon constraints and allow for slightly larger values of $\Delta A_{C P}$ we also consider model $10 \mu$, which is the same as model 10 with the lepton-charges ordered in such a way that the smallest ones are for muons, stressing the interplay between hadronic and leptonic sectors; model 10 can accommodate $\Delta A_{C P}^{\mathrm{NP}}$ up to $1.5 \times 10^{-3}$, while model $10 \mu$ can reach $1.8 \times 10^{-3}$. Figure 2 shows the stronger bound for each model, i.e., Eq. (39) for models 2, 5, 9 and $10 \mu$ (dash-dotted) and Eq. (38) for model 10 (dotted).

In Figs. 1 and 2 we show benchmark points. They pass constraints from $D$-mixing and semi(-muonic) decays, while giving $\Delta A_{C P}^{\mathrm{NP}} \sim 10^{-3}$. The golden star corresponds to model 2 with $\Delta F_{R}=12$ and

$\phi_{R} \sim \pi / 2, \quad g_{4} / M_{Z^{\prime}} \sim 0.38 / \mathrm{TeV}, \quad \theta_{u} \sim 1 \times 10^{-4}$.

The pink diamond corresponds to model $10 \mu$ with $\Delta F_{R}=$ 19 and

$\phi_{R} \sim \pi / 2, \quad g_{4} / M_{Z^{\prime}} \sim 2.3 / \mathrm{TeV}, \quad \theta_{u} \sim 1.7 \times 10^{-5}$.

We learn that $Z^{\prime}$-models with charges as in Table I can provide concrete NP-interpretations of $\Delta A_{C P}$ of the order of $10^{-3} \cdot D^{0}-\bar{D}^{0}$ mixing provides upper limits on the achievable $\Delta A_{C P}^{\mathrm{NP}}$. To distinguish the different model scenarios we explore correlations of $\Delta A_{C P}$ with other sectors, hadronic 2-body $D$-decays in Sec. IV and semileptonic $c \rightarrow u \ell^{+} \ell^{-}$transitions in Sec. V. 

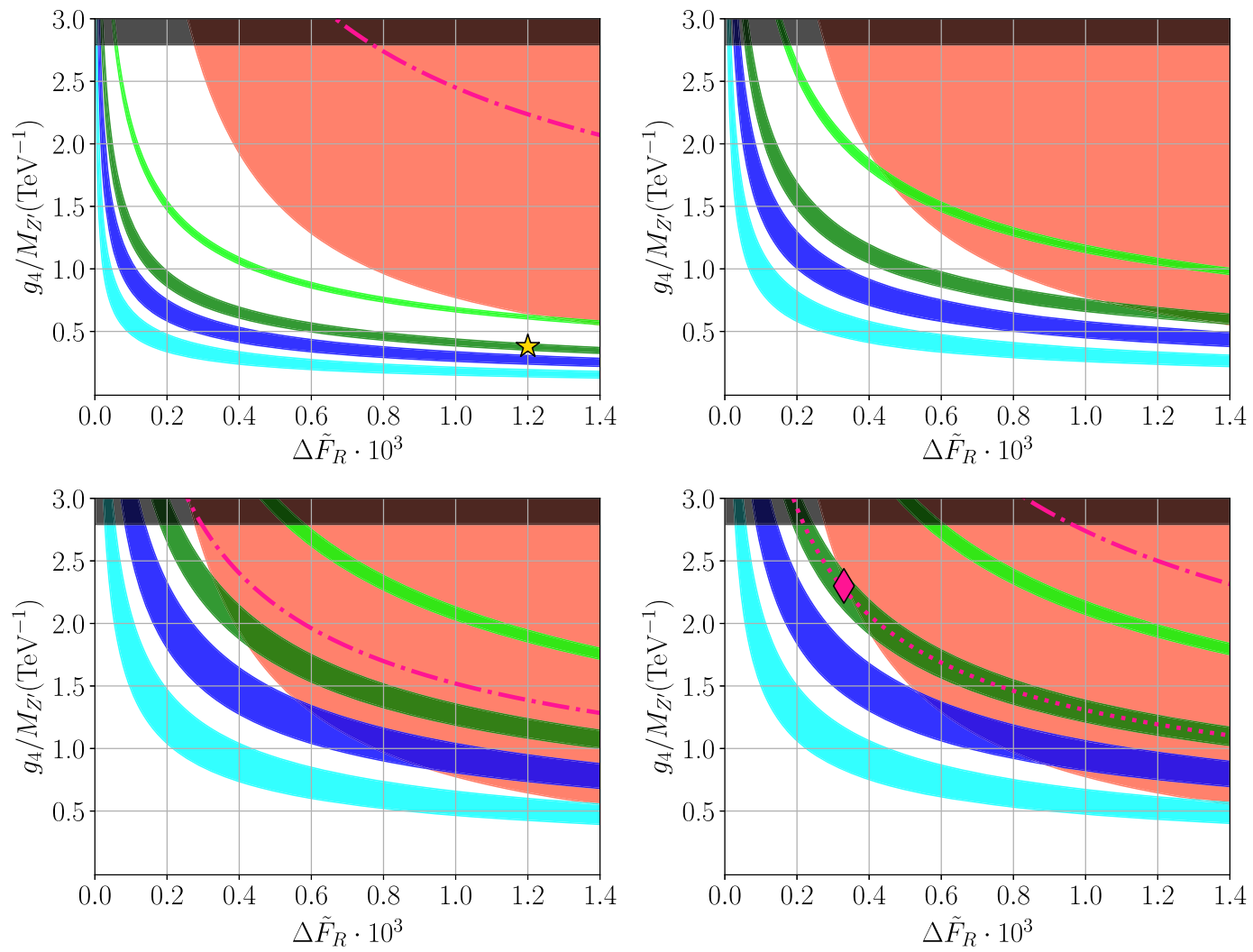

FIG. 2. $\left|\Delta A_{C P}^{\mathrm{NP}}\right|$ for different $Z^{\prime}$-models (2 upper left, 5 upper right, 9 lower left and $10(\mu)$ lower right) in the plane of $g_{4} / M_{Z^{\prime}}\left(\mathrm{TeV}^{-1}\right)$ and $\Delta \tilde{F}_{R}=\Delta F_{R} \cdot \theta_{u}$, together with the excluded region from $D^{0}-\bar{D}^{0}$ mixing (red). Light green, dark green, blue and cyan bands correspond to $\left|\Delta A_{C P}^{\mathrm{NP}}\right|=(4.0 \pm 0.2) \times 10^{-3},\left|\Delta A_{C P}^{\mathrm{NP}}\right|=(1.5 \pm 0.2) \times 10^{-3},\left|\Delta A_{C P}^{\mathrm{NP}}\right|=(8 \pm 2) \times 10^{-4}$ and $\left|\Delta A_{C P}^{\mathrm{NP}}\right|=(3 \pm 1) \times 10^{-4}$, respectively. The black region indicates the upper bound coming from perturbativity and direct searches in dimuon and dielectron spectra [27], which read $g_{4} \leq 4 \pi$ and $M_{Z^{\prime}} \geq 4.5 \mathrm{TeV}$, respectively. The magenta dash-dotted and dotted lines show the stronger (if any) of the bounds from Eqs. (38) and (39). In the lower right plot the dotted line corresponds to model 10, and the dash-dotted to model $10 \mu$. The golden star and pink diamond are benchmark points (40) and (41). See text for details.

\section{PATTERNS IN HADRONIC DECAYS}

$Z^{\prime}$-models with nonuniversal charges $F_{\psi}$ can give rise to large flavor-breaking effects which could explicitly violate relations between hadronic charm decays [28-31]. We study signatures of $Z^{\prime}$-induced U-spin and isospin breaking in Sec. IVA and Sec. IV B, respectively. $A_{C P}$ in $D^{0} \rightarrow \pi^{0} \pi^{0}$ is studied in Sec. IV C.

\section{A. U-spin patterns in $D^{0} \rightarrow \pi^{+} \pi^{-}, K^{+} K^{-}$}

U-spin breaking arises for $F_{Q_{1}} \neq F_{Q_{2}}$ or $F_{d_{1}} \neq F_{d_{2}}$, and can upset the U-spin sum rule [28]

$$
A_{C P}\left(D^{0} \rightarrow K^{+} K^{-}\right)+A_{C P}\left(D^{0} \rightarrow \pi^{+} \pi^{-}\right)=0 .
$$

To quantify deviations from this relation we define ${ }^{2}$

\footnotetext{
${ }^{2}$ For model $10(\mu)$ we use instead $\left|1+\frac{A_{C P}\left(D^{0} \rightarrow \pi^{+} \pi^{-}\right)}{A_{C P}\left(D^{0} \rightarrow K^{+} K^{-}\right)}\right|$to avoid $\mathrm{U}_{\text {break }}^{\text {tot }}>1$. It is tacitly understood that $K, Q_{2}, d_{2}$ and $\pi, Q_{1}, d_{1}-$ indices in Eq. (44) and following need to be swapped in this case.
}

$$
\mathrm{U}_{\text {break }}^{\text {tot }}=\left|1+\frac{A_{C P}\left(D^{0} \rightarrow K^{+} K^{-}\right)}{A_{C P}\left(D^{0} \rightarrow \pi^{+} \pi^{-}\right)}\right|
$$

In the $\mathrm{U}-$ spin limit $\mathrm{U}_{\text {break }}^{\text {tot }}=0$.

Using Eqs. (36), $\mathrm{U}_{\text {break }}^{\text {tot }}$ can be written as

$$
\mathrm{U}_{\text {break }}^{\text {tot }}=\left|1+\frac{c_{K} F_{Q_{2}}+d_{K} F_{d_{2}}}{c_{\pi} F_{Q_{1}}+d_{\pi} F_{d_{1}}}\right| .
$$

In Table III we give $\mathrm{U}_{\text {break }}^{\text {tot }}$ for models 2, 4, 5, 9 and $10(\mu)$, for $M_{Z^{\prime}}=6 \mathrm{TeV}$. The variation of $\mathrm{U}_{\text {break }}^{\text {tot }}$ with $M_{Z^{\prime}}$ in the range shown is within a few percent.

Taking advantage of the smallness of the parameters $d_{K, \pi}$ relative to $c_{K, \pi}$, we perform a Taylor expansion in Eq. (44) up to $\mathcal{O}\left(d_{K}, d_{\pi}\right)$ to qualitatively understand how U-spin breaking in our models emerges. This leads to

$$
\mathrm{U}_{\text {break }}^{\mathrm{tot}} \approx\left|1+\frac{c_{K}}{c_{\pi}}-\frac{c_{K} d_{\pi} F_{d_{1}}}{c_{\pi}^{2} F_{Q_{1}}}+\frac{d_{K} F_{d_{2}}}{c_{\pi} F_{Q_{1}}}\right|
$$


TABLE III. Values of $\beta_{9 / 10}^{\ell \ell}$ in $(\mathrm{TeV})^{-2}$ for $\ell=\mu, e$ and dimensionless $\beta_{\pi^{\prime}, 0}$ as defined in Eq. (63) and Eq. (55), respectively, as well as $\mathrm{U}_{\text {break }}^{\text {tot }}$ in Eq. (44), see footnote 2, for $M_{Z^{\prime}}=6 \mathrm{TeV}$.

\begin{tabular}{lrrrrrrr}
\hline \hline Model & \multicolumn{1}{c}{$\beta_{9}^{\mu \mu}$} & \multicolumn{1}{c}{$\beta_{10}^{\mu \mu}$} & \multicolumn{1}{c}{$\beta_{9}^{e e}$} & $\beta_{10}^{e e}$ & \multicolumn{1}{c}{$\beta_{\pi^{0}}$} & \multicolumn{1}{c}{$\beta_{\pi^{\prime}}$} & $\mathrm{U}_{\text {break }}^{\text {tot }}$ \\
\hline 2 & 0.57 & -0.57 & -0.68 & 0.68 & -0.02 & -0.02 & 0.42 \\
4 & -1.04 & -0.35 & 1.04 & 0.35 & -0.03 & -0.03 & 0.22 \\
5 & -0.67 & 0 & 0.67 & 0 & -0.10 & -0.09 & 0.32 \\
9 & -20.56 & -14.07 & 15.15 & -2.17 & -1.89 & -1.75 & 0.22 \\
10 & 37.25 & 3.39 & -32.73 & 1.13 & 1.31 & 1.22 & 0.91 \\
$10 \mu$ & -4.52 & -4.52 & -32.73 & 1.13 & 1.31 & 1.22 & 0.91 \\
\hline \hline
\end{tabular}

for $F_{Q_{1}}=F_{Q_{2}} \neq 0$ (models 2, 4 and 5), while for $F_{Q_{1}}=$ $F_{Q_{2}}=0$ (models 9 and $10(\mu)$ ) Eq. (44) simply becomes

$$
\mathrm{U}_{\text {break }}^{\text {tot }}=\left|1+\frac{d_{K} F_{d_{2}}}{d_{\pi} F_{d_{1}}}\right|
$$

For models with $F_{Q_{1}}=F_{Q_{2}} \neq 0$ different sources of $\mathrm{U}-$ spin breaking exist. The second term in Eq. (45) accounts for effects originating from interference between the SM amplitude and the $F_{Q_{1,2}}$-charges. This contribution is responsible for $22 \% \mathrm{U}$-spin breaking, which is of the same order of magnitude as the expected U-spin breaking uncertainty of the SM. In contrast, the last two terms in Eq. (45) are pure NP U-spin breaking effects. Equation (45) can further be simplified with $d_{K} \approx \frac{c_{K}}{c_{\pi}} d_{\pi}$ due to $\chi_{\pi} \approx \chi_{K}$, which holds numerically at the level of $\mathcal{O}(0.1-1) \%$. It follows that

$$
\mathrm{U}_{\mathrm{break}}^{\mathrm{tot}} \approx\left|1+\frac{c_{K}}{c_{\pi}}+\frac{d_{K}}{c_{\pi}}\left(\frac{F_{d_{2}}-F_{d_{1}}}{F_{Q_{1}}}\right)\right|
$$

highlighting that pure NP U-spin breaking effects are induced by

$$
\mathrm{U}_{\text {break }}^{\mathrm{NP}}=\frac{d_{K}}{c_{\pi}}\left|\frac{F_{d_{2}}-F_{d_{1}}}{F_{Q_{1}}}\right| \approx 0.04\left|\frac{F_{d_{2}}-F_{d_{1}}}{F_{Q_{1}}}\right|,
$$

which indicates how both the pion chiral enhancement and $r_{2}$ suppress U-spin breaking in these models. Therefore, values of $F_{d_{2}}-F_{d_{1}} \sim \mathcal{O}(1)$ such as in model 5, induce U-spin breaking within the range expected within the $\mathrm{SM} \lesssim 30 \%$. In model $4, F_{d_{i}}=0$ and $\mathrm{U}_{\text {break }}^{\mathrm{NP}}=0$, that is, U-spin breaking is SM-like. On the other hand, for $F_{d_{2}}-F_{d_{1}} \sim \mathcal{O}(10)$ as in model 2, large U-spin breaking effects can arise and would be discernible with future sensitivities for $A_{C P}\left(K^{+} K^{-}\right)$and $A_{C P}\left(\pi^{+} \pi^{-}\right)$shown in Table IV.

For models with $F_{Q_{1}}=F_{Q_{2}}=0$ we obtain for the pure NP U-spin breaking from Eq. (46)
TABLE IV. $\quad C P$ asymmetries and future sensitivities $\sigma$ in units of $10^{-4}$ at LHCb Run 1-3 (Run 1-5) [32] and Belle II with $50 \mathrm{ab}^{-1}$ [33].

\begin{tabular}{lccc}
\hline \hline$\times 10^{-4}$ & Data & $\sigma_{\text {LHCb }}$ & $\sigma_{\text {Belle II }}$ \\
\hline$\Delta A_{C P}$ & $-15.4 \pm 2.9[1]$ & $1.3(0.3)$ & $\ldots$ \\
$\Delta A_{C P}^{\mathrm{HFLAV}}$ & $-16.4 \pm 2.8[2]$ & $1.3(0.3)$ & $\ldots$ \\
$A_{C P}\left(D^{0} \rightarrow K^{+} K^{-}\right)$ & $-9 \pm 11[2]$ & $3(0.7)$ & 3 \\
$A_{C P}\left(D^{0} \rightarrow \pi^{+} \pi^{-}\right)$ & $-1 \pm 14[2]$ & $3(0.7)$ & 5 \\
$A_{C P}\left(D^{0} \rightarrow \pi^{0} \pi^{0}\right)$ & $-3 \pm 64[2]$ & $\ldots$ & 9 \\
$A_{C P}\left(D^{+} \rightarrow \pi^{+} \pi^{0}\right)$ & $+290 \pm 290 \pm 30[34]$ & $\ldots$ & 17 \\
\hline \hline
\end{tabular}

$$
\mathrm{U}_{\text {break }}^{\mathrm{NP}}\left(F_{Q_{1,2}}=0\right) \approx 0.78\left|\frac{F_{d_{2}}-F_{d_{1}}}{F_{d_{1}}}\right|
$$

which, unlike in Eq. (48), is unsuppressed. Models with $F_{Q_{1}}=F_{Q_{2}}=0$ are therefore prime candidates for sizable NP U-spin breaking effects. Models 9 and $10(\mu)$ have been constructed for this purpose. However, in model $9 F_{d_{2}}=$ $F_{d_{1}}$ and U-spin breaking arises from $d_{K} \neq-d_{\pi}$ only, and is SM-like.

Note, the strong phases associated with NP are assumed to be similar, $\sin \delta_{\pi} \simeq \sin \delta_{K}$, and order one; violation of Eq. (42) can be suppressed or even further enhanced by U-spin breaking in the strong phases. While this is an uncertainty on the NP interpretation, $Z^{\prime}$-signals could even be more striking.

In Figs. 3 and 4 we show the contributions of models 2, 5, 9 and $10(\mu)$ to the individual $C P$ asymmetries $A_{C P}\left(K^{+} K^{-}\right)$and $A_{C P}\left(\pi^{+} \pi^{-}\right)$in blue, magenta, yellow and cyan, respectively. The U-spin limit is given by the red dashed line with $30 \%$ U-spin breaking indicated by the

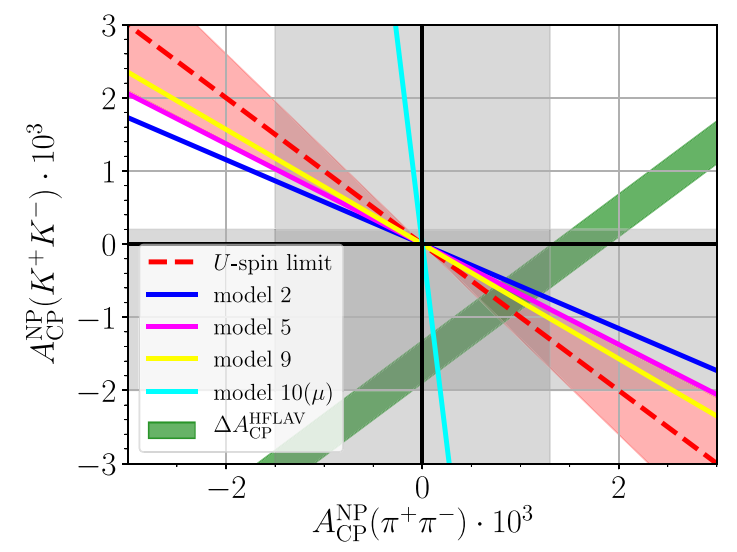

FIG. 3. $A_{C P}\left(K^{+} K^{-}\right)$versus $A_{C P}\left(\pi^{+} \pi^{-}\right)$with predictions in the $Z^{\prime}$-models $2,5,9$ and $10(\mu)$ in blue, magenta, yellow and cyan lines, respectively. The green band corresponds to the experimental world average of $\Delta A_{C P}$ (3) at $1 \sigma$. The gray bands indicate the present experimental $1 \sigma$ regions given in Table IV. The Uspin limit (42) (red dashed line) and $\lesssim 30 \%$ SM-like U-spin breaking (red area) is also shown. 

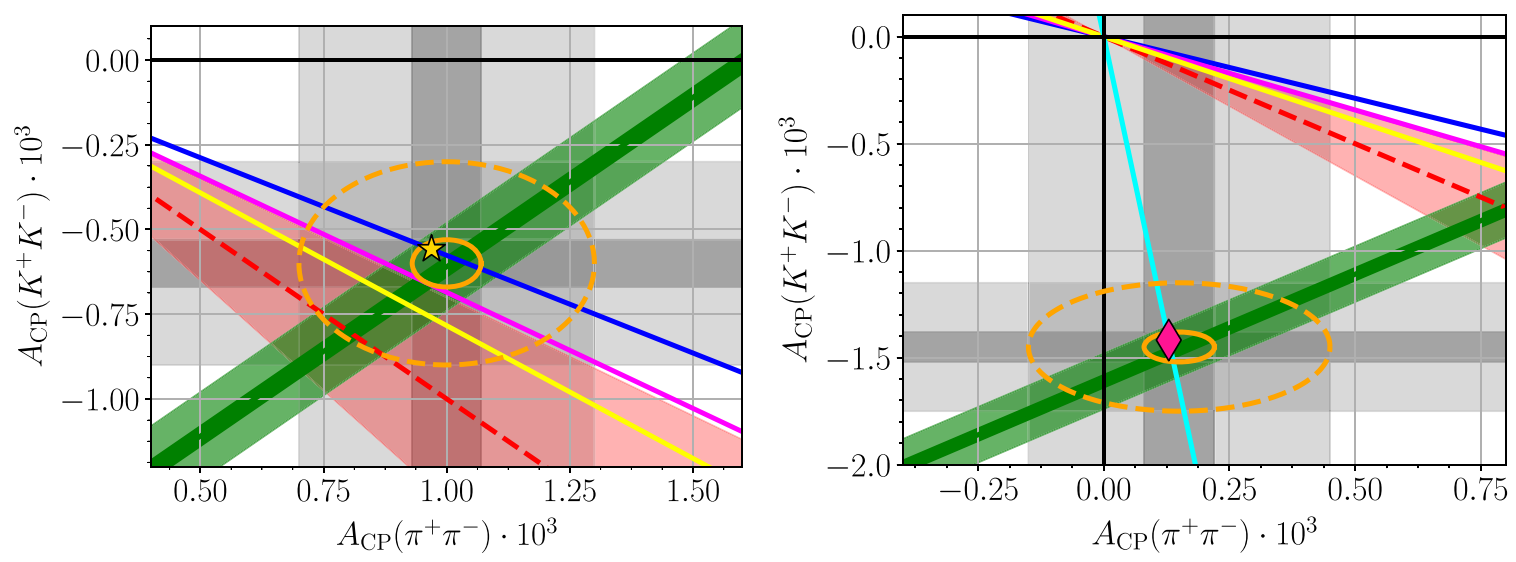

FIG. 4. Future projections for $A_{C P}\left(K^{+} K^{-}\right)$versus $A_{C P}\left(\pi^{+} \pi^{-}\right)$with predictions in the $Z^{\prime}$-models $2,5,9$ and $10(\mu)$ in blue, magenta, yellow and cyan lines, respectively. The green band corresponds to the central value of the present experimental world average of $\Delta A_{C P}$ (3) with future $1 \sigma$ sensitivities according to Table IV. The gray bands illustrate two future measurements of the individual asymmetries. The central values are given in Eq. (50), the uncertainties are scaled according to Table IV. Lighter (darker) bands correspond to LHCb Run 1-3 (1-5). Assuming for simplicity gaussian errors a dashed (solid) ellipse occurs around model 2 (plot to the left) and model $10 \mu$ (plot to the right) for LHCb Run 1-3 (1-5). The U-spin limit (42) (red dashed line) and $\lesssim 30 \%$ SM-like U-spin breaking (red area) is also shown. The golden star and pink diamond are benchmark points (40) and (41).

red contour. Present experimental bounds from Table IV are shown in Fig. 3 as $1 \sigma$ regions in gray for the individual asymmetries and in green for $\Delta A_{C P}$. The future sensitivities are indicated in light (dark) gray and green bands in Fig. 4 for LHCb Run 1-3 (1-5). We use the following central values for the plot to the left (right)

$$
\begin{aligned}
A_{C P}^{\mathrm{cen}}\left(K^{+} K^{-}\right) & =-0.6 \times 10^{-3}\left(-1.45 \times 10^{-3}\right), \\
A_{C P}^{\mathrm{cen}}\left(\pi^{+} \pi^{-}\right) & =1.0 \times 10^{-3}\left(0.15 \times 10^{-3}\right) .
\end{aligned}
$$

The orange error ellipses illustrate the NP sensitivity of the projected uncertainties of $A_{C P}\left(K^{+} K^{-}\right)$and $A_{C P}\left(\pi^{+} \pi^{-}\right)$ assuming no correlations. A future data-based analysis which takes into account correlations between the individual asymmetries and $\Delta A_{C P}$ can be expected to be more powerful.

U-spin symmetry within the SM is broken at the level of $30 \%$. We find that flavorful $Z^{\prime}$-models can exceed this by far (model $10(\mu)$ ), or moderately (model 2 ), which makes the measurements of $A_{C P}\left(K^{+} K^{-}\right)$and $A_{C P}\left(\pi^{+} \pi^{-}\right)$smoking guns for NP, within reach of Belle II and LHCb with the projected sensitivities.

\section{B. Isospin breaking patterns in $D^{+} \rightarrow \pi^{+} \pi^{0}$}

Isospin breaking arises in $Z^{\prime}$-models if $F_{u_{1}} \neq F_{d_{1}}$. In charm physics, the hadronic decay $D^{+} \rightarrow \pi^{+} \pi^{0}$ represents a formidable candidate to study these effects, because the $C P$ asymmetry $A_{C P}\left(\pi^{+} \pi^{0}\right)$, defined by

$$
A_{C P}\left(\pi^{+} \pi^{0}\right)=\frac{\Gamma\left(D^{+} \rightarrow f^{+}\right)-\Gamma\left(D^{-} \rightarrow f^{-}\right)}{\Gamma\left(D^{+} \rightarrow f^{+}\right)+\Gamma\left(D^{-} \rightarrow f^{-}\right)},
$$

with $f^{ \pm}=\pi^{ \pm} \pi^{0}$ is a clean SM null test [35].
Following the same procedure as in Sec. III D for $\Delta A_{C P}^{\mathrm{NP}}$ we obtain, using $\theta_{u} \ll 1$,

$$
A_{C P}^{\mathrm{NP}}\left(\pi^{+} \pi^{0}\right) \sim \frac{g_{4}^{2}}{M_{Z^{\prime}}^{2}} \theta_{u} \Delta F_{R} d_{\pi^{\prime}}\left(F_{d_{1}}-F_{u_{1}}\right)
$$

with

$$
d_{\pi^{\prime}}=-\frac{1}{a_{\pi^{\prime}}} r_{2}\left(m_{c}, M_{Z^{\prime}}\right)
$$

Here, $a_{\pi^{\prime}}$ denotes the tree-level contribution to $D^{+} \rightarrow \pi^{+} \pi^{0}$ whose modulus has been fixed experimentally, see Appendix A for details. Numerical values of $d_{\pi^{\prime}}$ for different values of $M_{Z^{\prime}}$ are given in Table II. Inserting Eq. (35) into Eq. (52), we obtain

$$
A_{C P}^{\mathrm{NP}}\left(\pi^{+} \pi^{0}\right) \sim \beta_{\pi^{\prime}} \cdot \Delta A_{C P}^{\mathrm{NP}},
$$

where

$$
\beta_{\pi^{\prime}}=\frac{d_{\pi^{\prime}}\left(F_{d_{1}}-F_{u_{1}}\right)}{c_{K} F_{Q_{2}}+d_{K} F_{d_{2}}-c_{\pi} F_{Q_{1}}-d_{\pi} F_{d_{1}}} .
$$

Values of $\beta_{\pi^{\prime}}$ for $M_{Z^{\prime}}=6 \mathrm{TeV}$ and different $Z^{\prime}$-models can be seen in Table III. Since we have lost information about the signs of the leading SM decay amplitudes with which NP is interfering, we cannot predict the relative sign between the $C P$ asymmetries in Eq. (54) without relying on assumptions on the strong interaction. Note, unlike for $A_{C P}\left(K^{+} K^{-}\right)$and $A_{C P}\left(\pi^{+} \pi^{-}\right)$, there is no SM flavor symmetry here at work. 
We find that model 9 and $10(\mu)$ induce values near

$$
A_{C P}^{\mathrm{NP}}\left(\pi^{+} \pi^{0}\right) \sim(1-2) \cdot \Delta A_{C P}^{\mathrm{NP}},
$$

which for $\Delta A_{C P}^{\mathrm{NP}} \sim 10^{-3}$ is within the projected sensitivity of Belle II with $50 \mathrm{ab}^{-1}$ [33], see Table IV. Models 2, 4 and 5 induce $A_{C P}^{\mathrm{NP}}\left(\pi^{+} \pi^{0}\right) \lesssim 0.1 \cdot \Delta A_{C P}^{\mathrm{NP}} \sim 10^{-4}$, beyond the reach of current facilities.

This behavior can be understood by expanding Eq. (55) in the $d_{i}$ up to $\mathcal{O}\left(d_{i}\right)$. For $F_{Q_{1}}=F_{Q_{2}}=0$ [model 9 and $10(\mu)$ ], we find that $\beta_{\pi^{\prime}}$ scales with $d_{\pi^{\prime}} / d_{K} \approx-1.6$ times a combination of charges $\left(F_{d_{1}}-F_{u_{1}}\right) / F_{d_{2}}(1+\cdots) \sim \mathcal{O}(1)$ resulting in $\mathcal{O}(1)$ isospin breaking effects. For models with $F_{Q_{1}}=F_{Q_{2}} \neq 0$ instead a suppression factor $d_{\pi^{\prime}} /\left(c_{K}-c_{\pi}\right) \approx 0.03$ exists from the chiral enhancement of the $(V-A) \times(V+A)$ operators, leading to $\beta_{\pi^{\prime}}$ of $\mathcal{O}\left(10^{-2}-10^{-1}\right)$.

$$
\text { C. } D^{\mathbf{0}} \rightarrow \pi^{0} \pi^{0}
$$

We work out the $C P$ asymmetry for $D^{0} \rightarrow \pi^{0} \pi^{0}$ decays because of its potential to diagnose patterns of NP [36]. In addition, the experimental prospect at Belle II for $A_{C P}\left(D^{0} \rightarrow \pi^{0} \pi^{0}\right)$ is about a factor of two better than for $A_{C P}\left(D^{+} \rightarrow \pi^{+} \pi^{0}\right)$, see Table IV. In the $Z^{\prime}$-models, $A_{C P}\left(D^{0} \rightarrow \pi^{0} \pi^{0}\right)$ is obtained from Eqs. (52) and (53) after replacing subscripts $\pi^{\prime}$ by $\pi^{0}$ with otherwise identical expressions. Therefore, with $\beta_{\pi^{0}}$ given in Table III,

$$
A_{C P}^{\mathrm{NP}}\left(\pi^{0} \pi^{0}\right) \sim \beta_{\pi^{0}} \cdot \Delta A_{C P}^{\mathrm{NP}},
$$

hence

$$
A_{C P}^{\mathrm{NP}}\left(\pi^{0} \pi^{0}\right) \lesssim 2 \cdot \Delta A_{C P}^{\mathrm{NP}},
$$

with the limit saturated by model 9 , and which is within the sensitivity of Belle II with $50 \mathrm{ab}^{-1}$ [33], see Table IV. Furthermore,

$$
\frac{A_{C P}^{\mathrm{NP}}\left(\pi^{0} \pi^{0}\right)}{A_{C P}^{\mathrm{NP}}\left(\pi^{+} \pi^{0}\right)} \sim \frac{\beta_{\pi^{0}}}{\beta_{\pi^{\prime}}}=\frac{a_{\pi^{\prime}}}{a_{\pi^{0}}}=1.08 \pm 0.10,
$$

holds universally for all $Z^{\prime}$-models with $F_{u_{1}} \neq F_{d_{1}}$. Experimental tests of Eq. (59) can support a $Z^{\prime}$ interpretation, however, additional uncertainties from large, unknown strong phases exist, which can modify the relation. As discussed after Eq. (54), we cannot predict the relative sign between the $C P$ asymmetries (57), (59) without relying on input on the strong interaction.

\section{SEMILEPTONIC DECAYS VS $\Delta A_{C P}$}

The dominant Wilson coefficients in $c \rightarrow u \ell^{+} \ell^{-}$ transitions are $C_{9 / 10}^{\ell \ell(\prime)}$, defined in Eq. (15). In flavorful $Z^{\prime}$-models [18]

$$
\begin{aligned}
& C_{9 / 10}^{\ell \ell}\left(M_{Z^{\prime}}\right)=-\frac{\pi}{\sqrt{2} G_{F} \alpha_{e}} \frac{g_{L}^{u c}}{M_{Z^{\prime}}^{2}}\left(g_{R}^{\ell \ell} \pm g_{L}^{\ell \ell}\right), \\
& C_{9 / 10}^{\ell \ell \rho}\left(M_{Z^{\prime}}\right)=-\frac{\pi}{\sqrt{2} G_{F} \alpha_{e}} \frac{g_{R}^{u c}}{M_{Z^{\prime}}^{2}}\left(g_{R}^{\ell \ell} \pm g_{L}^{\ell \ell}\right),
\end{aligned}
$$

where $g_{R}^{\ell \ell}=g_{4} F_{e_{i}}$ and $g_{L}^{\ell \ell}=g_{4} F_{L_{i}}$ with in general different couplings for muons and electrons. As explained in Sec. III C, we analyze in this work $Z^{\prime}$-models with $g_{L}^{u c}=0$ and $\operatorname{Im}\left(g_{R}^{u c}\right)$ large.

$C P$ asymmetries in the branching ratios are induced by interference of NP, here through $g_{R}^{u c}$, with $C_{9}^{\text {eff }}$, the effective coefficient of $O_{9}$ present in the SM, which is leptonuniversal, depends on the dilepton invariant mass and has sizable hadronic contributions and provides sizable strong phases. This interference term is sensitive to $C_{9}^{\ell \ell \prime}$ only. Angular analysis offers further opportunities. An interesting recent example for the latter is $D^{0} \rightarrow \pi^{+} \pi^{-} \mu^{+} \mu^{-}$decays $[21,22,37]$. Notably, the angular observables $I_{5,6,7}$ are GIM-protected in the SM and clean null tests [21]. In the $Z^{\prime}$-models under consideration, $I_{5,6}$ are induced by $\operatorname{Re}\left(C_{9}^{\ell \ell \prime} \cdot C_{10}^{\ell \ell * *}\right)$ and $\operatorname{Im}\left(C_{10}^{\ell \ell \prime} \cdot C_{9}^{\text {eff* }}\right)$, whereas $I_{7}$ is induced by $\left.\operatorname{Re}\left[\left(C_{9}^{\text {eff } *}-C_{9}^{\ell \ell \prime}\right) \cdot C_{10}^{\ell \ell / *}\right)\right]$. $C P$ asymmetries in angular asymmetries, on the other hand, can stem from naïve $T$-odd observables and do not rely on strong phases $\left(I_{7,8,9}\right)$. $C P$-odd ones $\left(I_{5,6,8,9}\right)$ provide $C P$ asymmetries that can be measured without tagging, see Ref. [21] for details. A complete and detailed analysis of angular asymmetries in $Z^{\prime}$-models is beyond the scope of this work. What we do want to point out here is that a global analysis of angular and $C P$ asymmetries can probe both $C_{9}^{\ell \ell \prime}$ and $C_{10}^{\ell \ell \prime}$ for electrons, $\ell=e$ and muons, $\ell=\mu$ separately, and therefore can distinguish different $U(1)^{\prime}-$ charge assignments.

Taking the imaginary part of Eq. (61) and employing Eq. (35), we obtain

$$
\operatorname{Im}\left(C_{9 / 10}^{\ell \ell \prime}\right) \sim \frac{\pi}{\sqrt{2} G_{F} \alpha_{e}} \beta_{9 / 10}^{\ell \ell} \cdot \Delta A_{C P}^{\mathrm{NP}},
$$

where

$$
\beta_{9 / 10}^{\ell \ell}=\frac{F_{e_{i}} \pm F_{L_{i}}}{c_{K} F_{Q_{2}}+d_{K} F_{d_{2}}-c_{\pi} F_{Q_{1}}-d_{\pi} F_{d_{1}}} .
$$

Values of $\beta_{9 / 10}^{\ell \ell}$ for $\ell=\mu$, $e$ in $(\mathrm{TeV})^{-2}$ are given in Table III. For $\Delta A_{C P}^{\mathrm{NP}} \sim 10^{-3}$ we find

$$
\operatorname{Im}\left(C_{9 / 10}^{\ell \ell \prime}\right) \sim 0.03(\mathrm{TeV})^{2} \cdot \beta_{9 / 10}^{\ell \ell},
$$

consistent with $C_{9 / 10}^{\ell \ell \prime}=\mathcal{O}\left(10^{-2}\right)$ for $g_{L}^{u c}=0, g_{R}^{u c} \neq 0$ [18] and for $\beta_{9 / 10}^{\ell \ell}=\mathcal{O}\left(1 / \mathrm{TeV}^{2}\right)$ (models 2, 4 and 5). Models 9 and $10(\mu)$ have sizable couplings to leptons, and in addition $F_{Q_{1,2}}=0$, which bring a factor of $c_{\pi, K} / d_{\pi, K}$, see Eq. (63), 
score $\beta_{9 / 10}^{\ell \ell}=\mathcal{O}\left(10 / \mathrm{TeV}^{2}\right)$ and sizable $C_{9 / 10}^{\ell \ell \prime}=\mathcal{O}\left(10^{-1}\right)$. As values of $\operatorname{Im}\left(C_{9 / 10}^{\ell \ell \prime}\right) \gtrsim \mathcal{O}\left(10^{-2}-10^{-1}\right)$ suffice to induce $C P$ asymmetries beyond the $\mathrm{SM}$ in semileptonic $D$-decays at the few percent level and above [13,18,21,23], all models can simultaneously lead to $\Delta A_{C P}^{\mathrm{NP}} \sim 10^{-3}$ with NP patterns in $c \rightarrow u \ell^{+} \ell^{-}$decays.

In Fig. 5 we show the imaginary part of Wilson coefficients with dielectrons (upper plots) and dimuons (lower plots) for different models as in Eq. (62). Plots to the left show lepton vector couplings versus lepton axial vector couplings, $\operatorname{Im}\left(C_{9}^{\ell \ell \prime}\right)$ vs $\operatorname{Im}\left(C_{10}^{\ell \ell \prime}\right)$, respectively. Also given is $\operatorname{Im}\left(C_{10}^{\ell \ell \prime}\right)=-\operatorname{Im}\left(C_{9}^{\ell \ell \prime}\right)$ (thin gray line). The lines corresponding to model 2,4 , and 5 end when the corresponding $\Delta A_{C P}^{\mathrm{NP}}$ exceeds $2 \times 10^{-3}$. Results are lepton nonuniversal as anticipated and sensitive to the lepton doublet and singlet charges. In the plots to the right the correlation (62) between $\operatorname{Im}\left(C_{9}^{\ell \ell \prime}\right)$ (solid) and $\operatorname{Im}\left(C_{10}^{\ell \ell \prime}\right)$ (dashed) and $\left|\Delta A_{C P}^{\mathrm{NP}}\right|$ in the $Z^{\prime}$-models 2, 9, 10 and $10 \mu$ is made explicit. Curves for models 4 and 5 are only in mild excess of those for model 2, or smaller, see Table III, and are not shown to avoid clutter.

As couplings to electrons and muons differ, lepton nonuniversality in charm $[18,21,38]$ is induced, for example in the ratio of branching ratios of $D \rightarrow \pi \mu^{+} \mu^{-}$and $D \rightarrow \pi e^{+} e^{-}$using identical kinematic cuts, $R_{\pi}^{D}$. To better control SM backgrounds from intermediate resonances $R=\phi, \eta^{(\prime)}, \rho, \ldots, \quad$ via $\quad D \rightarrow \pi R\left(\rightarrow \ell^{+} \ell^{-}\right)$, interesting regions are for low (high) dilepton mass, below the $\eta$-mass (above the $\phi$-mass), see Ref. [18] for details. We focus on the high mass region as it has fewer sensitivity to unknown strong phases from the resonances.

Using $\beta_{9 / 10}^{\ell \ell}$ from Table III and Eq. (64) we find that all models yield order one deviations from the universality limit $R_{\pi}^{D}=1$. Except for model $10 \mu$, which has smaller couplings to muons by construction, all models can induce significant enhancements or suppressions from the SM. In particular, in the high mass region, for $\phi_{R}=\pi / 2$ and varying strong resonance phases, see Ref. [18] for details,
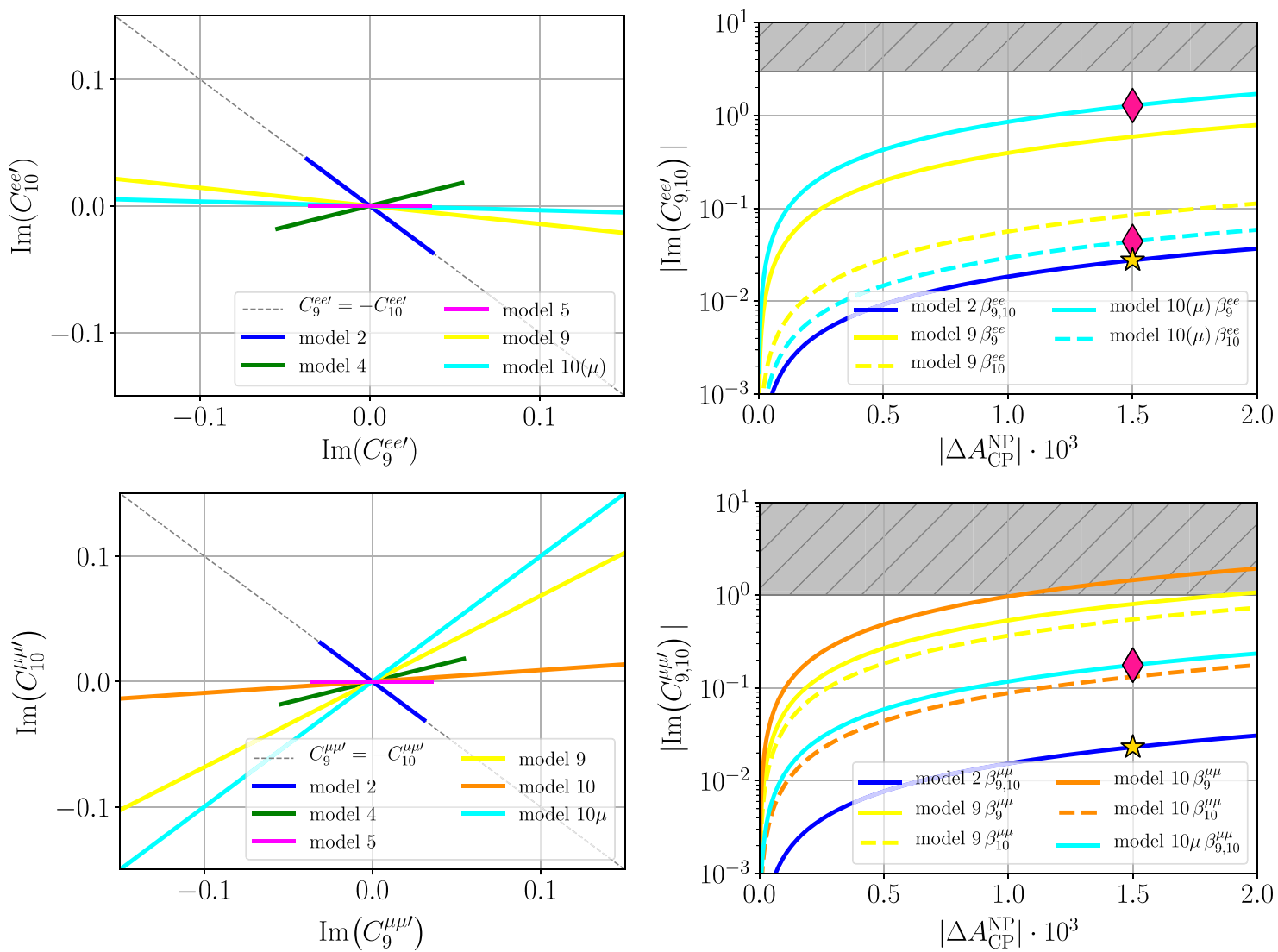

FIG. 5. The interplay between semi-electronic (upper plots) and semi-muonic (lower plots) charm FCNCs (62) and $\Delta A_{C P}$. The lines for model 2, 4 and 5 end when the corresponding $\Delta A_{C P}^{\mathrm{NP}}$ exceeds $2 \times 10^{-3}$ (plots to the left). In the plots to the right the correlation (62) between $\operatorname{Im}\left(C_{9}^{\ell \ell \prime}\right)$ (solid) and $\operatorname{Im}\left(C_{10}^{\ell \ell \prime}\right)$ (dashed) and $\left|\Delta A_{\mathrm{CP}}\right|$ in the $Z^{\prime}$-models 2, 9, 10 and $10 \mu$ is made explicit. The golden star and pink diamond are benchmark points (40) (model 2) and (41) (model 10 $\mu$ ), respectively. The shaded areas correspond to the upper limits (18). See text for details. 


$$
\begin{aligned}
R_{\pi}^{D} & \sim[0.6 \ldots 1.5] & & (\text { model } 2,4,5), \\
R_{\pi}^{D} & \sim[0.2 \ldots 70] & & (\text { model } 9), \\
R_{\pi}^{D} & \sim[0.2 \ldots 11] & & (\text { model } 10), \\
R_{\pi}^{D} & \sim[0.03 \ldots 0.8] & & (\text { model } 10 \mu),
\end{aligned}
$$

allowing to signal NP.

\section{CONCLUSIONS}

Patterns of observables are indispensable for pinning down an underlying NP-dynamics. We looked globally into hadronic and semileptonic charm decays and their respective $C P$ asymmetries. We find that there is strong benefit in doing so.

Most important, all flavorful, anomalyfree $Z^{\prime}$-models in Table I can simultaneously accommodate $\Delta A_{C P}^{\mathrm{NP}} \sim 10^{-3}$ and induce measurable $C P$ asymmetries in the semileptonic $c \rightarrow u \ell^{+} \ell^{-}$modes for $\ell=e$ or $\ell=\mu$ above the SM. An observation of $C P$ violation in, for instance, $D \rightarrow \pi \ell^{+} \ell^{-}$ or $D \rightarrow \pi \pi \ell^{+} \ell^{-}$decays supports a NP-interpretation of $\Delta A_{C P}$, Eqs. (1) and (3), see Fig. 5.

Additional cross checks are provided by $C P$ asymmetries in $D^{0} \rightarrow \pi^{+} \pi^{-}, D^{0} \rightarrow K^{+} K^{-}$, which probe for U-spin breaking NP, see Figs. 3 and 4 for present data and future sensitivities, respectively. In addition, isospin violating NP can be observed with projected sensitivities at Belle II in $D^{0} \rightarrow \pi^{0} \pi^{0}, D^{+} \rightarrow \pi^{+} \pi^{0}$ decays, whose $C P$ asymmetries can exceed $\Delta A_{C P}$, Eqs. (54) and (57).

In the $Z^{\prime}$-models lepton nonuniversality is generic, and observable in the ratio of branching fractions of $D \rightarrow$ $\pi \mu^{+} \mu^{-}$and $D \rightarrow \pi e^{+} e^{-}$decays, as briefly discussed in Sec. V. The $Z^{\prime}$-model 9 with order one enhancements over the universality limit, $R_{\pi}^{D} \gg 1$, also induces $A_{C P}^{\mathrm{NP}}\left(\pi^{+} \pi^{0}\right) \sim A_{C P}^{\mathrm{NP}}\left(\pi^{0} \pi^{0}\right) \lesssim 2 \cdot \Delta A_{C P}^{\mathrm{NP}} . Z^{\prime}$-model $10 \mu$ with order one suppression of the universality limit, $R_{\pi}^{D}<1$ exhibits sizable NP U-spin breaking $A_{C P}^{\mathrm{NP}}\left(\pi^{+} \pi^{-}\right) \ll$ $A_{C P}^{\mathrm{NP}}\left(K^{+} K^{-}\right) \sim \Delta A_{C P}$.

Checking correlations pins down models. Improved data and sensitivities from LHCb and Belle II are important in this program. We encourage and look forward to further $C P$ studies of rare semileptonic and hadronic charm decays.

\section{ACKNOWLEDGMENTS}

This work is supported by the Studienstiftung des Deutschen Volkes (MG) and the Bundesministerium für Bildung und Forschung-BMBF (HG).

\section{APPENDIX A: EXPERIMENTAL INPUT}

We extract the modulus of the dominant, SM decay amplitudes from data on branching ratios [27] given in Table V. We use
TABLE V. Measured branching ratios [27] and $a_{P}$-parameters from Eq. (A2) for different decay modes.

\begin{tabular}{lrc}
\hline \hline Mode & BR(mode) & \multicolumn{1}{c}{$a_{P}$} \\
\hline$D^{0} \rightarrow K^{+} K^{-}$ & $(4.08 \pm 0.06) \times 10^{-3}$ & $1.19 \pm 0.04$ \\
$D^{0} \rightarrow \pi^{+} \pi^{-}$ & $(1.455 \pm 0.024) \times 10^{-3}$ & $0.94 \pm 0.07$ \\
$D^{0} \rightarrow \pi^{0} \pi^{0}$ & $(8.26 \pm 0.25) \times 10^{-4}$ & $0.71 \pm 0.05$ \\
$D^{+} \rightarrow \pi^{0} \pi^{+}$ & $(1.247 \pm 0.033) \times 10^{-3}$ & $0.77 \pm 0.05$ \\
\hline \hline
\end{tabular}

$$
\operatorname{BR}\left(D \rightarrow P_{1} P_{2}\right)=\frac{\left|\mathcal{A}_{P}\right|^{2}}{16 \pi m_{D}} \sqrt{1-\frac{4 m_{P}^{2}}{m_{D}^{2}}} \tau_{D},
$$

where [39]

$$
\mathcal{A}_{P}=\eta_{P} \lambda_{P} a_{P} \frac{G_{F}}{\sqrt{2}}\left(m_{D}^{2}-m_{P}^{2}\right) f_{0}^{D \rightarrow P}\left(m_{P}^{2}\right) f_{P}
$$

$P=\pi, \pi^{0}, \pi^{\prime}, K, \lambda_{\pi}=\lambda_{d}$ and $\lambda_{K}=\lambda_{s}$ and

$$
\eta_{P}=\left\{\begin{array}{ll}
1 & P=\pi, \pi^{0}, K \\
\frac{1}{\sqrt{2}} & P=\pi^{\prime}
\end{array} .\right.
$$

The subscript $\pi^{\prime}$ corresponds to the $D^{+} \rightarrow \pi^{+} \pi^{0}$ channel, and $\pi^{0}$ to $D^{0} \rightarrow \pi^{0} \pi^{0}$. Relevant form factors $f_{0}^{D \rightarrow P}$ and decay constants $f_{P}$ are taken from Ref. [40] and [27], respectively. Resulting values of $a_{P}>0$ are given in Table V.

\section{APPENDIX B: EVOLUTION OF WILSON COEFFICIENTS}

The Wilson coefficients $\tilde{C}_{7,8,9,10}^{(\prime)}$ at the $Z^{\prime}$ mass scale (32) are evolved to the charm mass scale at LO in $\alpha_{s}$. The requisite anomalous dimension matrix for the operators $\tilde{Q}_{7,8,9,10}$ can be inferred from Ref. [41]. We obtain

$$
\gamma_{F}^{0}=\left(\begin{array}{cccc}
\frac{6}{N_{C}} & -6 & 0 & 0 \\
0 & \frac{6\left(1-N_{C}^{2}\right)}{N_{C}} & 0 & 0 \\
0 & 0 & \frac{-6}{N_{C}} & 6 \\
0 & 0 & 6 & \frac{-6}{N_{C}}
\end{array}\right),
$$

where $N_{C}=3$ is the number of colors. Since QCD conserves parity, $\gamma_{F}^{0}$ is identical for $\tilde{Q}_{i}$ and $\tilde{Q}_{i}^{\prime}$. Using Eq. (B1), the Wilson coefficients are evolved to the charm scale, integrating out degrees of freedom at the $\left(Z^{\prime}, t, b\right)$ scales,

$$
\vec{C}(\mu)=U_{4}\left(\mu, m_{b}\right) \hat{U}_{5}\left(m_{b}, m_{t}\right) \hat{U}_{6}\left(m_{t}, M_{Z^{\prime}}\right) \vec{C}\left(M_{Z^{\prime}}\right),
$$

where $\hat{U}_{f}\left(m_{1}, m_{2}\right) \equiv M_{f}\left(m_{1}\right) U_{f}\left(m_{1}, m_{2}\right)$ and $U_{f}\left(m_{1}, m_{2}\right)$ is the evolution matrix from scale $m_{2}$ to scale $m_{1}$ in an 
effective field theory with $f$ active flavors; $M_{f}$ is the threshold matrix that matches the two effective theories with $f-1$ and $f$ active flavors. At LO in $\alpha_{s}$, the $M_{f}$ matrices are equal to the identity matrix. For $\mu=m_{c}$ and $M_{Z^{\prime}}=6 \mathrm{TeV}$, one finds

$$
\begin{aligned}
& \tilde{C}_{7}^{(\prime)}\left(m_{c}\right)=0.829 \tilde{C}_{7}^{(\prime)}\left(M_{Z^{\prime}}\right), \\
& \tilde{C}_{8}^{(\prime)}\left(m_{c}\right)=1.224 \tilde{C}_{7}^{(\prime)}\left(M_{Z^{\prime}}\right)+4.502 \tilde{C}_{8}^{(\prime)}\left(M_{Z^{\prime}}\right), \\
& \tilde{C}_{9}^{(\prime)}\left(m_{c}\right)=1.404 \tilde{C}_{9}^{(\prime)}\left(M_{Z^{\prime}}\right)-0.718 \tilde{C}_{10}^{(\prime)}\left(M_{Z^{\prime}}\right), \\
& \tilde{C}_{10}^{(\prime)}\left(m_{c}\right)=-0.718 \tilde{C}_{9}^{(\prime)}\left(M_{Z^{\prime}}\right)+1.404 \tilde{C}_{10}^{(\prime)}\left(M_{Z^{\prime}}\right) .
\end{aligned}
$$

We use $m_{c}=(1.280 \pm 0.013) \mathrm{GeV}, m_{b}=(4.198 \pm 0.012) \mathrm{GeV}$ [42] and $m_{t}=(165.9 \pm 2.1) \mathrm{GeV}[43,44]$ and central values for the thresholds.

\section{APPENDIX C: HADRONIC MATRIX ELEMENTS}

In order to estimate the NP decay amplitudes, we need to determine the hadronic matrix elements for each operator given by Eqs. (24)-(31). For that purpose, we employ factorization of currents, $P=\pi, K$,

$$
\begin{aligned}
& \left\langle P^{+} P^{-}\left|Q_{i}\right| D^{0}\right\rangle \\
& \quad=\left\langle P^{+}\left|\left(\bar{q}_{1} \Gamma_{1} q_{2}\right)\right| 0\right\rangle\left\langle P^{-}\left|\left(\bar{q}_{3} \Gamma_{2} q_{4}\right)\right| D^{0}\right\rangle B_{i}^{P^{+} P^{-}},
\end{aligned}
$$

where $Q_{i}=\left(\bar{q}_{1} \Gamma_{1} q_{2}\right)\left(\bar{q}_{3} \Gamma_{2} q_{4}\right)$ is a 4-quark operator and $\Gamma_{1,2}$ represent possible Dirac and color structures while $q_{j}$ denote quarks. The factor $B_{i}^{P^{+} P^{-}}$parametrizes the deviation of the true hadronic matrix element from its naïve approximation, $\left.B_{i}^{P^{+} P^{-}}\right|_{\text {naïve }}=1$. For the NP contributions we work in this approximation. After employing Fierz identities in the flavor and color space, we find for $D^{0} \rightarrow K^{+} K^{-}$and $\pi^{+} \pi^{-}$decays

$$
\begin{gathered}
\left\langle\tilde{Q}_{7}\right\rangle_{K, \pi}=\frac{1}{N_{C}}\left\langle\tilde{Q}_{8}\right\rangle_{K, \pi}, \\
\left\langle\tilde{Q}_{8}\right\rangle_{K, \pi}=F_{d_{2}, d_{1}} \chi_{K, \pi}(\mu)\left\langle Q_{1}^{s, d}\right\rangle_{K, \pi}, \\
\left\langle\tilde{Q}_{9}\right\rangle_{K, \pi}=\frac{1}{N_{C}}\left\langle\tilde{Q}_{10}\right\rangle_{K, \pi}, \\
\left\langle\tilde{Q}_{10}\right\rangle_{K, \pi}=F_{Q_{2}, Q_{1}}\left\langle Q_{1}^{s, d}\right\rangle_{K, \pi},
\end{gathered}
$$

where $\langle\ldots\rangle_{P}=\left\langle P^{+} P^{-}|\ldots| D^{0}\right\rangle, \quad Q_{1}^{p}=(\bar{u} p)_{V-A}(\bar{p} c)_{V-A}$ and $\chi_{K, \pi}(\mu)$ are the usual chiral enhancements generated by $(V-A) \times(V+A)$ operators,

$$
\begin{aligned}
\chi_{K}(\mu) & =\frac{2 M_{K}^{2}}{m_{c}(\mu) m_{s}(\mu)}, \\
\chi_{\pi}(\mu) & =\frac{2 M_{\pi}^{2}}{m_{c}(\mu)\left(m_{d}+m_{u}\right)(\mu)},
\end{aligned}
$$

with values $\chi_{K}\left(m_{c}\right) \approx 3.626$ and $\chi_{\pi}\left(m_{c}\right) \approx 3.655$ at the charm mass scale. For the $\tilde{Q}_{i}^{\prime}$ operators the same relations hold but with the proper exchange of charges $F_{Q_{i}} \leftrightarrow F_{d_{i}}$.

For $D^{+} \rightarrow \pi^{0} \pi^{+}$decays we find

$$
\begin{gathered}
\left\langle\tilde{Q}_{7}\right\rangle_{\pi^{\prime}}=\frac{1}{N_{C}}\left\langle\tilde{Q}_{8}\right\rangle_{\pi^{\prime}}, \\
\left\langle\tilde{Q}_{8}\right\rangle_{\pi^{\prime}}=\frac{\chi_{\pi}(\mu)}{\sqrt{2}}\left(F_{u_{1}}-F_{d_{1}}\right)\left\langle Q_{1}^{u}\right\rangle_{u}, \\
\left\langle\tilde{Q}_{9}\right\rangle_{\pi^{\prime}}=\frac{1}{N_{C}}\left\langle\tilde{Q}_{10}\right\rangle_{\pi^{\prime}}=0,
\end{gathered}
$$

and for the corresponding $\tilde{Q}_{i}^{\prime}$ operators

$$
\begin{gathered}
\left\langle\tilde{Q}_{7}^{\prime}\right\rangle_{\pi^{\prime}}=\frac{1}{N_{C}}\left\langle\tilde{Q}_{8}^{\prime}\right\rangle_{\pi^{\prime}}=0, \\
\left\langle\tilde{Q}_{9}^{\prime}\right\rangle_{\pi^{\prime}}=\frac{1}{N_{C}}\left\langle\tilde{Q}^{\prime}{ }_{10}\right\rangle_{\pi^{\prime}}, \\
\left\langle\tilde{Q}^{\prime}{ }_{10}\right\rangle_{\pi^{\prime}}=\frac{1}{\sqrt{2}}\left(F_{u_{1}}-F_{d_{1}}\right)\left\langle Q_{1}^{u}\right\rangle_{u} .
\end{gathered}
$$

For $D^{0} \rightarrow \pi^{0} \pi^{0}$ decays we obtain

$$
\begin{gathered}
\left\langle\tilde{Q}_{7}\right\rangle_{\pi^{0}}=\frac{1}{N_{C}}\left\langle\tilde{Q}_{8}\right\rangle_{\pi^{0}}, \\
\left\langle\tilde{Q}_{8}\right\rangle_{\pi^{0}}=\frac{\chi_{\pi}(\mu)}{2}\left(F_{u_{1}}-F_{d_{1}}\right)\left\langle Q_{1}^{u}\right\rangle_{u}, \\
\left\langle\tilde{Q}_{9}\right\rangle_{\pi^{0}}=\frac{1}{N_{C}}\left\langle\tilde{Q}_{10}\right\rangle_{\pi^{0}}=0,
\end{gathered}
$$

and for the corresponding $\tilde{Q}_{i}^{\prime}$ operators

$$
\begin{gathered}
\left\langle\tilde{Q}_{7}^{\prime}\right\rangle_{\pi^{0}}=\frac{1}{N_{C}}\left\langle\tilde{Q}_{8}^{\prime}\right\rangle_{\pi^{0}}=0, \\
\left\langle\tilde{Q}_{9}^{\prime}\right\rangle_{\pi^{0}}=\frac{1}{N_{C}}\left\langle\tilde{Q}_{10}^{\prime}\right\rangle_{\pi^{0}}, \\
\left\langle\tilde{Q}^{\prime}{ }_{10}\right\rangle_{\pi^{0}}=\frac{1}{2}\left(F_{u_{1}}-F_{d_{1}}\right)\left\langle Q_{1}^{u}\right\rangle_{u},
\end{gathered}
$$

where $\langle\ldots\rangle_{\pi^{\prime}}=\left\langle\pi^{+} \pi^{0}|\ldots| D^{+}\right\rangle,\langle\ldots\rangle_{\pi^{0}}=\left\langle\pi^{0} \pi^{0}|\ldots| D^{0}\right\rangle$ and $\langle\ldots\rangle_{q}=\left\langle\bar{q} q|\ldots| D^{+}\right\rangle$. Equations (C2)-(C18) are obtained in the isospin limit, $m_{u}=m_{d}$ and $e=0$, since these isospin breaking corrections from within the SM are negligible with respect to the NP ones, $F_{u_{i}, d_{i}, Q_{i}} \neq 0$. 


\section{APPENDIX D: RGE FUNCTIONS}

Equation (35) for $\Delta A_{C P}^{\mathrm{NP}}$ accounts for the running and mixing of operators through the functions $r_{1,2}$. The latter can be obtained from the evolution of the Wilson coefficients described in Appendix B. We obtain

$$
r_{1}\left(m_{c}, M_{Z^{\prime}}\right)=\frac{R^{-2}}{3 \sqrt{2} G_{F} \lambda_{s}},
$$

$$
r_{2}\left(m_{c}, M_{Z^{\prime}}\right)=\frac{2 R^{1 / 2}-R^{-1}}{3 \sqrt{2} G_{F} \lambda_{s}}
$$

where

$$
R=\left(\frac{\alpha_{s}^{(4)}\left(m_{b}\right)}{\alpha_{s}^{(4)}\left(m_{c}\right)}\right)^{\frac{12}{25}}\left(\frac{\alpha_{s}^{(5)}\left(m_{t}\right)}{\alpha_{s}^{(5)}\left(m_{b}\right)}\right)^{\frac{12}{23}}\left(\frac{\alpha_{s}^{(6)}\left(M_{Z^{\prime}}\right)}{\alpha_{s}^{(6)}\left(m_{t}\right)}\right)^{\frac{4}{7}}
$$

[1] R. Aaij et al. (LHCb Collaboration), Phys. Rev. Lett. 122, 211803 (2019).

[2] Y.S. Amhis et al. (HFLAV Collaboration), arXiv: 1909.12524.

[3] M. Chala, A. Lenz, A. V. Rusov, and J. Scholtz, J. High Energy Phys. 07 (2019) 161.

[4] A. Dery and Y. Nir, J. High Energy Phys. 12 (2019) 104.

[5] F. Buccella, A. Paul, and P. Santorelli, Phys. Rev. D 99, 113001 (2019).

[6] H. N. Li, C. D. Lü, and F. S. Yu, arXiv:1903.10638.

[7] A. Soni, arXiv:1905.00907.

[8] H. Y. Cheng and C. W. Chiang, Phys. Rev. D 100, 093002 (2019).

[9] A. Khodjamirian and A. A. Petrov, Phys. Lett. B 774, 235 (2017).

[10] A. L. Kagan and L. Silvestrini, arXiv:2001.07207.

[11] U. Nierste, arXiv:2002.06686.

[12] A. Pich, Proc. Sci., LHCP2019 (2019) 078 [arXiv: 1911.06211].

[13] S. Fajfer and N. Košnik, Phys. Rev. D 87, 054026 (2013).

[14] J. Ellis, M. Fairbairn, and P. Tunney, Eur. Phys. J. C 78, 238 (2018).

[15] B. C. Allanach, J. Davighi, and S. Melville, J. High Energy Phys. 02 (2019) 082.

[16] J. Rathsman and F. Tellander, Phys. Rev. D 100, 055032 (2019).

[17] D. B. Costa, B. A. Dobrescu, and P. J. Fox, Phys. Rev. Lett. 123, 151601 (2019).

[18] R. Bause, M. Golz, G. Hiller, and A. Tayduganov, Eur. Phys. J. C 80, 65 (2020).

[19] J. Aebischer, A. J. Buras, M. Cerdà-Sevilla, and F. De Fazio, J. High Energy Phys. 02 (2020) 183.

[20] D. Choudhury, K. Deka, T. Mandal, and S. Sadhukhan, arXiv:2002.02349.

[21] S. De Boer and G. Hiller, Phys. Rev. D 98, 035041 (2018).

[22] R. Aaij et al. (LHCb Collaboration), Phys. Rev. Lett. 121, 091801 (2018).

[23] S. de Boer and G. Hiller, Phys. Rev. D 93, 074001 (2016).
[24] J. Fuentes-Martin, A. Greljo, J. Martin Camalich, and J. D. Ruiz-Alvarez, arXiv:2003.12421.

[25] B. Holdom, Phys. Lett. 166B, 196 (1986).

[26] W. Altmannshofer, R. Primulando, C. T. Yu, and F. Yu, J. High Energy Phys. 04 (2012) 049.

[27] M. Tanabashi et al. (Particle Data Group), Phys. Rev. D 98, 030001 (2018).

[28] Y. Grossman, Z. Ligeti, and D. J. Robinson, J. High Energy Phys. 01 (2014) 066.

[29] Y. Grossman and S. Schacht, J. High Energy Phys. 07 (2019) 020.

[30] Y. Grossman and S. Schacht, Phys. Rev. D 99, 033005 (2019).

[31] S. Müller, U. Nierste, and S. Schacht, Phys. Rev. Lett. 115, 251802 (2015).

[32] R. Aaij et al. (LHCb Collaboration), arXiv:1808.08865.

[33] E. Kou et al. (Belle-II Collaboration), Prog. Theor. Exp. Phys. (2019), 123C01; (2020), 029201.

[34] H. Mendez et al. (CLEO Collaboration), Phys. Rev. D 81, 052013 (2010).

[35] Y. Grossman, A. L. Kagan, and J. Zupan, Phys. Rev. D 85, 114036 (2012).

[36] G. Hiller, M. Jung, and S. Schacht, Phys. Rev. D 87, 014024 (2013).

[37] L. Cappiello, O. Cata, and G. D’Ambrosio, J. High Energy Phys. 04 (2013) 135.

[38] S. Fajfer and N. Košnik, Eur. Phys. J. C 75, 567 (2015).

[39] Y. Grossman, A. L. Kagan, and Y. Nir, Phys. Rev. D 75, 036008 (2007).

[40] V. Lubicz, L. Riggio, G. Salerno, S. Simula, and C. Tarantino (ETM Collaboration), Phys. Rev. D 96, 054514 (2017); 99, 099902(E) (2019).

[41] G. Buchalla, A. J. Buras, and M. E. Lautenbacher, Rev. Mod. Phys. 68, 1125 (1996).

[42] S. Aoki et al. (Flavour Lattice Averaging Group), Eur. Phys. J. C 80, 113 (2020).

[43] J. Fuster, A. Irles, D. Melini, P. Uwer, and M. Vos, Eur. Phys. J. C 77, 794 (2017).

[44] G. Aad et al. (ATLAS Collaboration), J. High Energy Phys. 10 (2015) 121. 